\title{
Model Reduction by Moment Matching: Beyond Linearity A Review of the Last 10 Years
}

\author{
Alessandro Astolfi, Giordano Scarciotti, Joel Simard, Nicolás Faedo and John V. Ringwood
}

\begin{abstract}
We present a review of some recent contributions to the theory and application of nonlinear model order reduction by moment matching. The tutorial paper is organized in four parts: 1) Moments of Nonlinear Systems; 2) Playing with Moments: Time-Delay, Hybrid, Stochastic, Data-Driven and Beyond; 3) The Loewner Framework; 4) Applications to Optimal Control and Wave Energy Conversion.
\end{abstract}

\section{INTRODUCTION}

Dynamic models are essential for analysis, estimation, and control of modern systems and physical behaviors. Unfortunately, high-fidelity requirements dictate an increase in dimensionality and complexity of such models and require powerful computational platforms. This has maintained the computational needs at the top or over the available possibilities. A possible approach to mitigate such problem is provided by the use of reduced order models. The model reduction problem can be informally formulated as the problem of finding a simplified description of a dynamical system in specific operating conditions, preserving at the same time specific properties. For linear systems the problem has been addressed from several viewpoints. The additional difficulties of the reduction of nonlinear systems carry the need to develop different or "enhanced" techniques.

Aim of this tutorial paper is to survey model reduction methods for nonlinear systems which rely on the notion of moment and to demonstrate, via a specific application, that moments provide a powerful tool to formulate and solve complex control problems using numerical efficient algorithms. While we acknowledge that there are several methods to construct reduced order models, for linear and nonlinear systems, and these have been widely studied, the focus of the paper is on a specific method, and we refer the reader to the literature, for example using [1] as a pointer for linear systems and [2] as a pointer for nonlinear systems.

A fundamental preliminary result for the development of model reduction by moment matching for nonlinear systems has been to recognize that the problem of determining the moments of a system corresponds to the problem of solving a particular Sylverster equation, see [3] and [4]. Exploiting this property, the notion of moment has been revisited for linear

Alessandro Astolfi, Giordano Scarciotti and Joel Simard are with the Department of Electrical and Electronic Engineering, Imperial College London, London SW7 2AZ, U.K. (e-mail: [a.astolfi,g.scarciotti,j.simard18]@ic.ac.uk).

Alessandro Astolfi is also with the Department of Civil Engineering and Computer Science Engineering, University of Rome "Tor Vergata," 00133 Rome, Italy.

Nicolás Faedo and John. V. Ringwood are with the Centre for Ocean Energy Research, Maynooth University, Co. Kildare, Ireland (e-mail: john.ringwood@mu.ie). systems and extended to nonlinear systems firstly in [5] and then in [6], [7], [8]. The method presented in this tutorial paper is based on the framework pioneered in [8] which has been extended in multiple directions and has contributed to a number of applications, see for example [9], [10]. On the basis of the definition of moment given in [8], it has been shown, under suitable assumptions, in [11] that the Loewner framework of [12] can be viewed as a special case of a two-sided moment-matching procedure. Hence the Loewner framework presents an alternative method for leftand right-sided interpolation, that is for matching twice as many moments of the underlying system as the moments that can be matched with the result in [8]. Note that, to date, a nonlinear enhancement of the Loewner framework has not yet been fully developed. Enhancements for some mild classes of nonlinearities have been given in [13], [14], and [15].

The rest of the paper is divided into four parts. The notion of moment for nonlinear systems is introduced in Section II, which is then followed by Section III in which such a notion is developed to time-delay, hybrid, and stochastic systems and the basic ideas of data-driven model reductions are described. Section IV introduces the so-called Loewner framework for model reduction of nonlinear systems, while the application of the notion of moment to optimal control problems, and in particular to problems arising in wave energy conversion, is discussed in Section V.

Notation. We use standard notation. $\mathbb{C}_{<0}\left(\mathbb{C}_{\geq 0}\right)$ denotes the set of complex numbers with negative (non-negative) real part. $\mathbb{R}_{<0}\left(\mathbb{R}_{>0}\right)$ denotes the set of negative (positive) real numbers. The symbol $I$ denotes the identity matrix and $\sigma(A)$ denotes the spectrum of the matrix $A \in \mathbb{R}^{n \times n}$. The symbol $|v|$, with $v \in \mathbb{R}^{n}$, indicates the Euclidean norm of the vector $v$. The vectorization of a matrix $A \in \mathbb{R}^{n \times m}$, denoted by vec $(A)$, is the $n m \times 1$ vector obtained by stacking the columns of the matrix $A$ one on top of the other, namely $\operatorname{vec}(A)=\left[a_{1}^{\top}, a_{2}^{\top}, \ldots, a_{m}^{\top}\right]^{\top}$, where $a_{i} \in \mathbb{R}^{n}$ is the $i$-th column of $A$ and the superscript $\top$ denotes the transposition operator. The convolution between two functions $f$ and $g$ over the set $\Omega \subset \mathbb{R}$, i.e. $\int_{\Omega} f(\tau) g(t-\tau) d \tau$ is denoted as $f * g$. The symbol $\otimes$ indicates the Kronecker product, whereas the symbol $\oplus$ indicates the direct sum. Given two functions, $f: Y \rightarrow Z$ and $g: X \rightarrow Y$, with $f \circ g: X \rightarrow Z$ we denote the composite function $(f \circ g)(x)=f(g(x))$ which maps all $x \in X$ to $f(g(x)) \in Z$.

\section{Moments of Nonlinear Systems}

The point of departure for the theory of model reduction by moment matching is the notion of moment. Moments have 
been traditionally defined in terms of the value of a function of a complex variable, for example the transfer function of a linear (not-necessarily finite-dimensional) system, at a regular point of the function.

Unfortunately, the class of systems that can be described using transfer functions is fairly limited. Hence, extending the notion of moments to nonlinear systems requires an alternative, albeit equivalent in the linear case, definition. To achieve this objective, we initially re-interprete the notion of moment for linear systems using a state-space formulation, that is we consider a linear, single-input, single-output ${ }^{1}$, continuous-time system described by equations of the form

$$
\begin{aligned}
& \dot{x}=A x+B u, \\
& y=C x,
\end{aligned}
$$

with $x(t) \in \mathbb{R}^{n}, u(t) \in \mathbb{R}, y(t) \in \mathbb{R}$, together with its associated transfer function

$$
W(s)=C(s I-A)^{-1} B .
$$

The following assumptions hold throughout the paper whenver we refer to linear systems.

Assumption 1. The triple of matrices $(A, B, C)$ is a minimal realization, that is the underlying linear system is reachable and observable.

Definition 1. [1] The 0-moment of system (1) at $s^{\star} \in \mathbb{C}$ is the complex number

$$
\eta_{0}\left(s^{\star}\right)=C\left(s^{\star} I-A\right)^{-1} B .
$$

The $k$-moment of the system (1) at $s^{\star} \in \mathbb{C}$ is the complex number

$$
\begin{aligned}
\eta_{k}\left(s^{\star}\right) & =\frac{(-1)^{k}}{k !}\left[\frac{d^{k}}{d s^{k}}\left(C(s I-A)^{-1} B\right)\right]_{s=s^{\star}} \\
& =C\left(s^{\star} I-A\right)^{-(k+1)} B
\end{aligned}
$$

Remark 1. While moments are classically defined only at regular points of the transfer function (2), the paper [16] has shown that the definition can be extended to singular points.

The following result provides the sought after alternative definition.

Lemma 1. [3], [8] Consider the system (1) and $s^{\star} \in \mathbb{C}$. Suppose $s^{\star} \notin \sigma(A)$. Then the moments $\eta_{0}\left(s^{\star}\right), \ldots, \eta_{k}\left(s^{\star}\right)$ are in one-to-one relation with the matrix $C \Pi$, where $\Pi$ is the (unique) solution of the Sylvester equation

$$
A \Pi+B L=\Pi S,
$$

with $S$ any non-derogatory ${ }^{2}$ real matrix such that

$$
\operatorname{det}(s I-S)=\left(s-s^{\star}\right)^{k+1},
$$

and $L$ such that the pair $(L, S)$ is observable.

\footnotetext{
${ }^{1}$ Similar considerations can be performed for multi-input, multi-output systems.

${ }^{2} \mathrm{~A}$ matrix is non-derogatory if its characteristic and minimal polynomials coincide.
}

This characterization lends itself to a natural nonlinear enhancement. As a matter of fact the equation (3) reveals that the cascaded system

$$
\begin{aligned}
\dot{\omega} & =S \omega, \\
\dot{x} & =A x+B L \omega
\end{aligned}
$$

has a well-defined invariant set described by the equation $x=\Pi \omega$ and the restriction of the system (5) to the invariant set is a copy of the system $\dot{\omega}=S \omega$. This means that one could define the moment of the system (1) at $\sigma(S)$ (with all eigenvalues counted with their multiplicity) by means of a specific invariant set (in the case of linear systems it is a subspace). This point of view leads naturally to the following developments.

Consider a nonlinear, single-input, single-output, continuous-time system described by equations of the form ${ }^{3}$

$$
\begin{aligned}
\dot{x} & =f(x, u), \\
y & =h(x),
\end{aligned}
$$

with $x(t) \in \mathbb{R}^{n}, u(t) \in \mathbb{R}, y(t) \in \mathbb{R}$, a signal generator described by the equations

$$
\begin{aligned}
\dot{\omega} & =s(\omega), \\
\theta & =l(\omega),
\end{aligned}
$$

with $\omega(t) \in \mathbb{R}^{\nu}$ and $\theta(t) \in \mathbb{R}$ and the interconnected system

$$
\begin{aligned}
\dot{\omega} & =s(\omega), \\
\dot{x} & =f(x, l(\omega)), \\
y & =h(x) .
\end{aligned}
$$

Suppose, in addition, that $f(0,0)=0, s(0)=0, l(0)=0$ and $h(0)=0$. The signal generator captures the requirement that one is interested in studying the behaviour of system (6) only in specific circumstances. However, for this to make sense and to provide a generalization of the notion of moment, we need the following assumptions and definitions.

Assumption 2. There is a unique mapping $\pi(\omega)$, locally defined in a neighborhood of $\omega=0$, which solves the partial differential equation

$$
f(\pi(\omega), l(\omega))=\frac{\partial \pi}{\partial \omega} s(\omega) .
$$

Assumption 2 implies that the interconnected system (8) possesses an invariant manifold, described by the equation $x=\pi(\omega)$. Note that the (well-defined) dynamics of the system restricted to the invariant manifold are described by $\dot{\omega}=s(\omega)$, i.e. are a copy of the dynamics of the signal generator (7).

Assumption 3. The signal generator (7) is observable and excitable [17].

Definition 2. Consider system (6) and the signal generator (7). Suppose Assumptions 2 and 3 hold. The function $h(\pi(\cdot))$, with $\pi(\cdot)$ solution of equation (9), is the moment of system (6) at $s(\cdot)$.

\footnotetext{
${ }^{3}$ All functions and mapping are sufficiently smooth.
} 
The above definitions allow to derive a connection between moments and the steady-state output response.

Theorem 1. Consider system (6) and the signal generator (7). Assume Assumption 3 holds. Assume the zero equilibrium of the system $\dot{x}=f(x, 0)$ is locally exponentially stable and system (7) is Poisson stable.

Then the moment of system (6) at $s(\cdot)$ coincides with the (locally well-defined) steady-state response of the output of the interconnected system (8).

To obtain an existence condition in the case in which the underlying system does not have an exponentially stable equilibrium at the origin and/or the signal generator is not Poisson stable one has to resort to a different approach, which relies on a nonlinear enhancement of the non-resonant condition which guarantees existence of the solution of the Sylvester equation (3). This approach, and the corresponding technicalities, see e.g. Definition 7, are detailed in Section IV.

With the definition of moment at hand, one can introduce the notion of reduced order model and characterize the solution of the model reduction problem by moment matching.

Definition 3. The system

$$
\dot{\xi}=\phi(\xi, u), \quad \psi=\kappa(\xi),
$$

with $\xi(t) \in \mathbb{R}^{\nu}$, is a model at $s(\cdot)$ of system (6) if system (10) has the same moment at $s(\cdot)$ as (6). In this case, system (10) is said to match the moment of system (6) at $s(\cdot)$. Furthermore, system (10) is a reduced order model of system (6) if $\nu<n$.

Theorem 2. Consider the system (6), the system (10) and the signal generator (7). Suppose Assumptions 2 and 3 hold. System (10) matches the moments of (6) at $s(\cdot)$ if the equation

$$
\phi(p(\omega), l(\omega))=\frac{\partial p}{\partial \omega} s(\omega)
$$

has a unique solution $p(\omega)$ such that

$$
h(\pi(\omega))=\kappa(p(\omega))
$$

where $\pi(\cdot)$ is the solution of equation (9).

The above result allows constructing a parameterized family of reduced order models. To this end, note that to construct a reduced order model it is necessary to determine mappings $\phi(\cdot, \cdot), \kappa(\cdot)$ and $p(\cdot)$ such that equations (11) and (12) hold, where $\pi(\omega)$ is the solution of equation (9).

Consider now the following assumption.

Assumption 4. There exists mappings $\kappa(\cdot)$ and $p(\cdot)$ such that $k(0)=0, p(0)=0, p(\omega)$ is locally differentiable, equation (12) holds and

$$
\operatorname{det} \frac{\partial p(\omega)}{\partial \omega}(0) \neq 0,
$$

i.e. the mapping $p(\cdot)$ possesses a local inverse $p^{-1}(\cdot)$.

Remark 2. Assumption 4 holds selecting $p(\omega)=\omega$ and $k(\omega)=h(\pi(\omega))$.
A direct computation shows that a family of reduced order models, all achieving moment matching, provided equation (11) has a solution $p(\cdot)$, is described by

$$
\begin{aligned}
\dot{\xi} & =\phi_{0}(\xi)+\frac{\partial p(\omega)}{\partial \omega} \phi_{1}(\xi) u, \\
\psi & =\kappa(\xi),
\end{aligned}
$$

where $\kappa(\cdot)$ and $p(\cdot)$ are such that Assumption 4 holds,

$$
\phi_{1}(\xi)=\tilde{\phi}_{1}\left(p^{-1}(\xi)\right),
$$

where $\tilde{\phi}_{1}(\cdot)$ is a free mapping, and

$$
\phi_{0}(\xi)=\left[\frac{\partial p(\omega)}{\partial \omega}\left(s(\omega)-\phi_{1}(p(\omega)) l(\omega)\right)\right]_{\omega=p^{-1}(\xi)} .
$$

In particular, selecting $p(\omega)=\omega$ yields the family of reduced order models described by

$$
\begin{aligned}
\dot{\xi} & =s(\xi)-\phi_{1}(\xi) l(\xi)+\phi_{1}(\xi) u, \\
\psi & =h(\pi(\xi))
\end{aligned}
$$

where $\phi_{1}(\cdot)$ is a free mapping.

It is possible to use the free parameter $\phi_{1}(\cdot)$ to achieve specific properties of the reduced order model. For example, model reduction by moment matching with a stability constraint can be achieved selecting, if possible, the free mapping $\phi_{1}(\cdot)$ such that the zero equilibrium of the system

$$
\dot{z}=s(z)-\phi_{1}(z) l(z)
$$

is locally asymptotically stable. This is possible, for example, if the pair

$$
\left(\frac{\partial l(\omega)}{\partial \omega}(0), \frac{\partial s(\omega)}{\partial \omega}(0)\right)
$$

is observable, or detectable. Note, however, that this is not necessary.

Note that, in general, the computation of moments and the solution of the model reduction problem requires the solution of a partial differential equation. This is however not the case if one is interested in the model reduction problem with 0 -moment matching at $s^{\star}=0$. Such a problem can be solved, under specific assumptions, without the need to solve any partial differential equation, as detailed in the following statement.

Proposition 1. Consider system (6) and the signal generator $\dot{\omega}=0, \theta=\omega$. Assume the zero equilibrium of the system $\dot{x}=f(x, 0)$ is locally exponentially stable. Then the zero moment of system (6) is (locally) well defined and given by $h(\pi(\cdot))$, with $\pi(\cdot)$ the unique solution of the algebraic equation $f(\pi(\omega), \omega)=0$. Finally, a reduced order model, for which the zero equilibrium is locally asymptotically stable is given by

$$
\dot{\xi}=-\phi_{1}(\xi)(\xi-u), \quad \psi=h(\pi(\xi)),
$$

with $\phi_{1}(\xi)$ such that $\phi_{1}(0)>0$.

We conclude this preliminary section with a few observations. The notion of moment expressed in Definition 2 has led to extensive investigations in the last decade. While some 
of these are summarized in the remainder of this tutorial, it is worth noting the development of a dual theory in which the signal generator is driven by the system the moment of which are to be computed [18]; the definition of moments for nonlinear time-delay systems [19] and for systems driven by infinite dimensional generators [20]; the solution of structure/mode preserving model reduction problems [21], [22]; the definition of a nonlinear enhancement of the notion of phasors applicable to the study of circuits with switched mode power electronic components [23]; and a fully datadriven approach [24], [25].

\section{Playing With Moments: Time-Delay, Hybrid, STOCHASTIC, DATA-DRIVEN AND BEYOND}

This part of the tutorial has two objectives. The first is to provide a guessing technique to determine the moments of general systems. The second is to provide a numerical method to compute the moments of nonlinear systems.

\section{A. Introduction}

It should be clear by now that the model reduction method presented in this paper relies on the solution of two equations. The first is an invariance equation that defines the moments, e.g. (3) or (9). The second is a moment matching condition that imposes that the moments of the system and of the reduced order model are identical e.g. (12). Consider now a system $\mathcal{S}_{x, u}^{y}$ described by the generic evolution law

$$
\mathcal{S}_{x, u}^{y}: \quad \sigma x=\mathcal{F}(x, u), \quad y=\mathcal{H}(x),
$$

where $x$ is the state, $u$ is the input and $y$ is the output. $\sigma$ is a generic operator that may indicate a derivative, a jump, a differential and so on. $\mathcal{F}$ and $\mathcal{H}$ are generic maps that may apply time-delays, describe a hybrid dynamics, a stochastic dynamics and so on. The only requirement that we impose on (14) is that the state $x$ satisfies the superposition principle. We refer to (14) as "any linear system". Moreover, if (14) describes a time-delay system, then we say that we are in the "time-delay framework"; if (14) describes a hybrid system, then we say "hybrid framework", and so on. We call the framework of Section II, the "standard framework".

Let $\mathcal{S}_{\omega}^{u}$ be any linear signal generator with state $\omega$, no input and output $u$ and $\mathcal{S}_{x, u}^{y}$ be any linear system with state $x$, input $u$ and output $y$. We indicate with

$$
\mathcal{S}_{\omega}^{u} \stackrel{u=L \omega}{\rightarrow} \mathcal{S}_{x, u}^{y}
$$

the interconnected system in which the input $u$ of $\mathcal{S}_{x, u}^{y}$ is given by $u=L \omega$, where $L$ is a vector of the right dimension. Under framework-dependent assumptions the interconnection (15) will have a well-defined steady-state $x^{s s}$ which is described by a relation of the type

$$
x^{s s}=\mathcal{P} \cdot \omega,
$$

where $\mathcal{P}$ is a framework-dependent object, i.e. it may be a constant matrix, a function, a stochastic process and so on (the symbol - indicates matrix to vector multiplication and can be omitted). Then the "moments of any linear system
$\mathcal{S}_{x, u}^{y}$ at any linear signal generator $\mathcal{S}_{\omega}^{u}$ " are the elements of the vector

$$
\mathcal{H} \circ \mathcal{P}
$$

where $\mathcal{P}$ is such that the invariance equation

$$
\sigma(\mathcal{P} \cdot \omega)=\mathcal{F}(\mathcal{P} \cdot \omega, L \omega)
$$

holds for all $\omega$.

We now show that a simple guessing method which allows extending the characterization of moments to any new linear framework consists of:

1) making a guess for $\mathcal{P}$ in (16);

2) and writing down equation (18).

Remark 3. What follows are not proofs that the moments in each of the analysed frameworks are defined in the way that we find using this guessing technique. The objective of the tutorial is different; namely it is to show that making a guess for (16) and using the relation (18) is a powerful technique to quickly find the moment-defining equation in a new framework. It turns out that these guesses are correct, as shown through additional work in each of the corresponding works cited in the following.

Remark 4. Despite calling all the frameworks of this section "linear", we point out that traditionally the term linear refers only to the standard framework. Thus, this part of the tutorial is "beyond linearity" in the sense that we are beyond the standard framework.

Remark 5. The same guessing technique can be applied to any nonlinear system. We restrict ourselves to linear systems for simplicity and to save space. We refer the reader to the cited works for the nonlinear versions of most of the results presented herein.

Citations to the works where each of these results were first developed are provided in the titles of the sections. A unified reference is the monograph [2].

\section{B. Time-delay systems [19]}

Consider a linear, single-input, single-output, continuoustime, time-delay system with constant delays ${ }^{4}$ described by the equations

$$
\begin{aligned}
E \dot{x}\left(t-\tau_{E}\right) & =A x\left(t-\tau_{A}\right)+B u\left(t-\tau_{B}\right), \\
y(t) & =C x\left(t-\tau_{C}\right),
\end{aligned}
$$

with $x(t) \in \mathbb{R}^{n}, u(t) \in \mathbb{R}, y(t) \in \mathbb{R}, E \in \mathbb{R}^{n \times n}, A \in \mathbb{R}^{n \times n}$, $B \in \mathbb{R}^{n \times 1}, C \in \mathbb{R}^{1 \times n}, \tau_{E} \in \mathbb{R}_{\geq 0}, \tau_{A} \in \mathbb{R}_{\geq 0}, \tau_{B} \in \mathbb{R}_{\geq 0}$ and $\tau_{C} \in \mathbb{R}_{\geq 0}$. We also assume that $x$ and $u$ are equipped with the required initial histories. Consider a signal generator described by the equations

$$
\dot{\omega}=S \omega, \quad u=L \omega,
$$

\footnotetext{
${ }^{4}$ The same procedure can be applied to distributed delays and multiple delays.
} 
with $\omega(t) \in \mathbb{R}^{\nu}, S \in \mathbb{R}^{\nu \times \nu}, L \in \mathbb{R}^{1 \times \nu}$. In this framework, we expect that the steady state $x^{s s}$ of $x$ obeys (if it exists) a relation of the type

$$
x^{s s}(t)=\Pi \omega(t),
$$

where $\Pi \in \mathbb{R}^{n \times \nu}$. Then by applying the guessing technique we obtain the following result.

Theorem 3. Assume the guess (21) is correct. Then the matrix $\Pi$ is the unique solution of the equation

$$
A \Pi e^{-S \tau_{A}}-E \Pi S e^{-S \tau_{E}}=-B L e^{-S \tau_{B}} .
$$

Moreover, then the moments of system (19) at the signal generator (20) are the elements of $C \Pi e^{-S \tau_{C}}$.

Proof. Note that $\dot{\omega}(t)=S \omega(t)$ with $\omega\left(t_{0}\right)=\omega_{0}$ has solution $\omega(t)=e^{S\left(t-t_{0}\right)} \omega\left(t_{0}\right)$. Consider now $t_{0}=t-\tau$. Then $\omega(t)=$ $e^{S \tau} \omega(t-\tau)$, which implies $\omega(t-\tau)=e^{-S \tau} \omega(t)$. Since at steady state we assume $x(t)=\Pi \omega(t)$, it follows that

$$
x(t-\tau)=\Pi \omega(t-\tau)=\Pi e^{-S \tau} \omega(t) .
$$

Moreover $\dot{x}(t-\tau)=\Pi \dot{\omega}(t-\tau)=\Pi S \omega(t-\tau)=$ $\Pi S e^{-S \tau} \omega(t)$. Thus equation (18) in this framework becomes

$$
E \Pi S e^{-S \tau_{E}} \omega(t)=A \Pi e^{-S \tau_{A}} \omega(t)+B L e^{-S \tau_{B}} \omega(t) .
$$

As this must hold for all $\omega$, the first statement follows. Similarly, noticing that $y^{s s}=C x^{s s}\left(t-\tau_{C}\right)=C \Pi e^{-S \tau_{C}} \omega(t)$, we obtain the form of (17).

\section{Systems in explicit form [23], [20], [26], [27]}

Consider again the linear system (1), but now we consider a periodic signal generator described by the equation

$$
\begin{aligned}
& \omega(t)=\Lambda(t) \omega_{0}, \quad u=L \omega, \\
& \Lambda(t)=\Lambda(t-T), \quad t \geq T,
\end{aligned}
$$

with $\omega(t) \in \mathbb{R}^{\nu}, u(t) \in \mathbb{R}, L \in \mathbb{R}^{1 \times \nu}, \Lambda(t) \in \mathbb{R}^{\nu \times \nu}$ such that $\Lambda(0)=I$, and $T \in \mathbb{R}_{>0}$ the period of the signal $u$. Note that (23) provides a very general class of models which contains the implicit model $\dot{\omega}=S \omega$ (when periodic), but that can describe several other signal generators. For instance, it can represent signals generated by a time-varying system of the form

$$
\dot{\omega}=S(t) \omega, \quad u=L \omega,
$$

with $S(t) \in \mathbb{R}^{\nu \times \nu}$, in which case $\Lambda$ is the transition matrix associated to (24).

Equation (23) can also represent a signal generator described by some classes of hybrid systems of the form

$$
\begin{array}{rll}
\dot{\omega}(t, k) & =S \omega(t, k), & u_{c}=L_{c} \omega, \\
\omega(t, k+1) & =J \omega(t, k), & u_{d}=L_{d} \omega,
\end{array}
$$

with $J \in \mathbb{R}^{\nu \times \nu}, L_{c} \in \mathbb{R}^{1 \times \nu}, L_{d} \in \mathbb{R}^{1 \times \nu}, u_{c}(t) \in \mathbb{R}$ and $u_{d}(t) \in \mathbb{R}$, which jumps and flows on some hybrid time domain $\mathbb{R} \times \mathbb{Z}$. In general, note that any periodic signal that can be written as the product of a function of time "times" the initial condition can be described by (23). For instance, if we consider a square wave $\Pi(t)$, then $\Pi(t)$ can be generated by a nonlinear system, i.e $\Lambda(t)=\operatorname{sign}(\sin (t))$ or by the hybrid system (25), i.e $\Lambda(t)=J^{\left\lfloor\frac{t}{T}\right\rfloor} e^{S t}$. It is evident that the characterization of the moments for the explicit signal generator (23) would solve the problem of model reduction by moment matching for many different classes of input signals, not necessarily linear.

In this framework, we expect that the steady state $x^{s s}$ of $x$ obeys (if it exists) a relation of the type

$$
x^{s s}(t)=\Pi(t) \omega(t),
$$

where $\Pi(t) \in \mathbb{R}^{n \times \nu}$ is a time-varying matrix. Then by applying the guessing technique we obtain the following result.

Theorem 4. Assume the guess (26) is correct. Then the matrix $\Pi(t)$ is defined by

$$
\Pi(t)=\left(I-e^{A T}\right)^{-1}\left[\int_{t-T}^{t} e^{A(t-\tau)} B L \Lambda(\tau) d \tau\right] \Lambda(t)^{-1} .
$$

Moreover, then the moments of system (1) at the signal generator (23) are the elements of $C \Pi(t)$.

Proof. The solution of system (1) is $x(t)=e^{A\left(t-t_{0}\right)} x\left(t_{0}\right)+$ $\int_{t_{0}}^{t} e^{A(t-\tau)} B u(\tau) d \tau$. Using the change of variable $t_{0}=t-T$, the guess $x^{s s}(t)=\Pi(t) \omega(t)$, and recalling that $\Lambda(t)=$ $\Lambda(t-T)$ yields

$$
\begin{aligned}
\Pi(t) \Lambda(t) \omega\left(t_{0}\right)= & e^{A T} \Pi(t-T) \Lambda(t) \omega\left(t_{0}\right)+ \\
& +\int_{t-T}^{t} e^{A(t-\tau)} B L \Lambda(\tau) \omega\left(t_{0}\right) d \tau .
\end{aligned}
$$

Since the input to (1) is periodic, so is its steady-state. This implies that $\Pi(t)=\Pi(t-T)$. Now, since the previous equation must hold for any $\omega\left(t_{0}\right)$, we obtain

$$
\left(I-e^{A T}\right) \Pi(t)=\int_{t-T}^{t} e^{A(t-\tau)} B L \Lambda(\tau) \Lambda(t)^{-1} d \tau,
$$

from which (27) follows.

If $\mathcal{T} \subset \mathbb{R}_{>0}$ is an interval of time on which $\Lambda$ is differentiable, then $\Pi(t)$ solves the equation

$$
\dot{\Pi}=A \Pi+B L-\Pi \dot{\Lambda} \Lambda^{-1},
$$

for all $t \in \mathcal{T}$. The proof is omitted for reasons of space, see [2] for a complete discussion.

\section{Stochastic systems [28]}

Consider a linear, single-input, single-output, continuoustime, stochastic system described by the equations

$d x_{t}=\left(A x_{t}+B u_{t}\right) d t+\left(F x_{t}+G u_{t}\right) d \mathcal{W}_{t}, \quad y_{t}=C x_{t}$,

with $A \in \mathbb{R}^{n \times n}, B \in \mathbb{R}^{n \times 1}, F \in \mathbb{R}^{n \times n}, G \in \mathbb{R}^{n \times 1}$ and $C \in \mathbb{R}^{1 \times n}$. Here $x_{t}$ is a stochastic process defined on some probability space $(\Omega, \mathcal{G}, \mathbb{P})$ where $\Omega$ is the set of events, $\mathcal{F}$ is a $\sigma$-algebra on $\Omega$ and $\mathbb{P}$ is a probability measure on the measurable space $(\Omega, \mathcal{G}) . \mathcal{W}_{t}$ indicates a standard Wiener process defined on $(\Omega, \mathcal{G}, \mathbb{P})$. All stochastic differential equations are interpreted with the Itô convention. 
Consider a signal generator described by the equations

$$
d \omega_{t}=S \omega_{t} d t+J \omega_{t} d \mathcal{W}_{t}, \quad u_{t}=L \omega_{t},
$$

with $S \in \mathbb{R}^{\nu \times \nu}, J \in \mathbb{R}^{\nu \times \nu}$ and $L \in \mathbb{R}^{1 \times \nu}$.

In this framework, we expect that the steady state $x^{s s}$ of $x$ obeys (if it exists) a relation of the type

$$
x_{t}^{s s}=\mathcal{X}_{t} \omega_{t},
$$

where $\mathcal{X}_{t} \in \mathbb{R}^{n \times \nu}$ is a stochastic process. Then by applying the guessing technique we obtain the following result.

Theorem 5. Assume the guess (30) is correct. Then the matrix $\mathcal{X}_{t}$ is the steady-state solution of the equation

$$
\begin{aligned}
d \mathcal{X}_{t}= & \left(A \mathcal{X}_{t}-\mathcal{X}_{t}\left(S-J^{2}\right)-F \mathcal{X}_{t} J+B L-G L J\right) d t \\
& +\left(F \mathcal{X}_{t}-\mathcal{X}_{t} J+G L\right) d \mathcal{W}_{t},
\end{aligned}
$$

namely

$\mathcal{X}_{t}=\Phi_{t}\left[\int_{-\infty}^{t} \Phi_{\tau}^{-1}(B L-F G L) \Sigma_{\tau} d \tau+\int_{-\infty}^{t} \Phi_{\tau}^{-1} G L \Sigma_{\tau} d \mathcal{W}_{\tau}\right] \Sigma_{t}^{-1}$,

where $\Phi_{t}$ is the fundamental matrix of the homogeneous equation corresponding to (28). Moreover, the moments of system (28) at the signal generator (28) are the elements of $C \mathcal{X}_{t}$.

Proof. Using the stochastic product rule we have

$$
\begin{aligned}
d\left(\mathcal{X}_{t} \omega_{t}\right) & =d \mathcal{X}_{t} \omega_{t}+\mathcal{X}_{t} d \omega_{t}+d \mathcal{X}_{t} d \omega_{t} \\
& =d \mathcal{X}_{t} \omega_{t}+\mathcal{X}_{t}\left(S \omega_{t} d t+J \omega_{t} d \mathcal{W}_{t}\right)+\left[\mathcal{X}_{t}\right]_{\mathcal{W}} J \omega_{t} d t
\end{aligned}
$$

where the notation $\left[\mathcal{X}_{t}\right]_{\mathcal{W}}$ indicates the diffusion coefficient of $d \mathcal{X}_{t}$ (the component which multiplies $d \mathcal{W}_{t}$ ). On the other hand

$$
d x_{t}^{s s}=\left(A \mathcal{X}_{t}+B L\right) \omega_{t} d t+\left(F \mathcal{X}_{t}+G L\right) \omega_{t} d \mathcal{W}_{t} .
$$

Since the previous two equations must hold for any $\omega_{t}$, we obtain

$$
\begin{aligned}
& d \mathcal{X}_{t}+\left[\mathcal{X}_{t}\right]_{\mathcal{W}} J d t \\
& \quad=\left(A \mathcal{X}_{t}-\mathcal{X}_{t} S+B L\right) d t+\left(F \mathcal{X}_{t}-\mathcal{X}_{t} J+G L\right) d \mathcal{W}_{t}
\end{aligned}
$$

from which it follows that $\left[\mathcal{X}_{t}\right]_{\mathcal{W}}=F \mathcal{X}_{t}-\mathcal{X}_{t} J+G L$, thus proving (31). We omit the derivation of (32) for reasons of space, see [28] for a complete proof.

\section{E. On-line moment estimation from data [24], [25]}

Solving equation (9) with respect to the mapping $\pi$ is a difficult task even when there is perfect knowledge of the dynamics of the system, i.e. the mapping $f$. When $f$ is not known, equation (9) may be solved numerically requiring information on the state of the system. In practice, only measurements of the output $y$ may be available which further complicates the task of determining $\pi$. In this section we develop an algorithm to determine the moment of nonlinear systems using measurements of the output. Note that all the results of the chapter exploit the relation between moments and steady-state response.
Recall that under the assumptions of Theorem 1, the equation

$$
y(t)=h(\pi(\omega(t)))+\varepsilon(t),
$$

where $\varepsilon(t)$ is an exponentially decaying signal, holds. We introduce the following standard assumption.

Assumption 5. The mapping $h \circ \pi$ belongs to the function space identified by the family of continuous basis functions $\varphi_{j}: \mathbb{R}^{\nu} \rightarrow \mathbb{R}$, with $j=1, \ldots, M(M$ may be $\infty)$, i.e. there exist $\Gamma_{j} \in \mathbb{R}$, with $j=1, \ldots, M$, such that

$$
h(\pi(\omega))=\sum_{j=1}^{M} \Gamma_{j} \varphi_{j}(\omega),
$$

for any $\omega$.

In other words, we are assuming that the mapping $h \circ \pi$ can be decomposed as a weighted sum of basis functions. To determine the family of basis functions in Assumption 5 we can implement a trial and error procedure or use prior knowledge (e.g. use functions belonging to the same class as the ones generated by the signal generator).

Let

$$
\begin{aligned}
\Gamma & =\left[\begin{array}{llll}
\Gamma_{1} & \Gamma_{2} & \ldots & \Gamma_{N}
\end{array}\right], \\
\Omega(\omega(t)) & =\left[\begin{array}{llll}
\varphi_{1}(\omega(t)) & \varphi_{2}(\omega(t)) & \ldots & \varphi_{N}(\omega(t))
\end{array}\right]^{\top},
\end{aligned}
$$

with $N \leq M$. Using a weighted sum of basis functions, equation (33) can be written as

$y(t)=\sum_{j=1}^{N} \Gamma_{j} \varphi_{j}(\omega(t))+e(t)+\varepsilon(t)=\Gamma \Omega(\omega(t))+e(t)+\varepsilon(t)$,

where $e(t)=\sum_{N+1}^{M} \Gamma_{j} \varphi_{j}(\omega(t))$ is the truncation error resulting by cutting the vectors in $\Gamma \Omega$ at $N$. Let $\Gamma_{k}$ be an on-line estimate of the matrix $\Gamma$ computed at $T_{k}^{w}=$ $\left\{t_{k-w+1}, \ldots, t_{k-1}, t_{k}\right\}$ with $0 \leq t_{0}<t_{1}<\cdots<t_{k-w}<$ $\cdots<t_{k}<\cdots<t_{q}$, with $w>0$ and $q \geq w$, namely computed at the time $t_{k}$ using the last $w$ instants of time $t_{i}$ and assuming that $e(t)$ and $\epsilon(t)$ are known. Since this last assumption does not hold in practice, consider the approximation

$$
y(t) \approx \sum_{j=1}^{N} \widetilde{\pi}_{j} \varphi_{j}(\omega(t))=\widetilde{\Gamma} \Omega(\omega(t)),
$$

which neglects the truncation error $e(t)$ and the transient error $\varepsilon(t)$. Let $\widetilde{\Gamma}_{k}=\left[\begin{array}{llll}\widetilde{\pi}_{1} & \widetilde{\pi}_{2} & \ldots & \widetilde{\pi}_{N}\end{array}\right]$ be the approximation, in the sense of (34), of the estimate $\Gamma_{k}$. Let $\widetilde{U}_{k} \in \mathbb{R}^{w \times N}$ and $\widetilde{\Upsilon}_{k} \in \mathbb{R}^{w}$, with $w \geq \nu$, be time-snapshots defined as

$$
\widetilde{U}_{k}=\left[\begin{array}{llll}
\Omega\left(\omega\left(t_{k-w+1}\right)\right) & \ldots & \Omega\left(\omega\left(t_{k-1}\right)\right) & \Omega\left(\omega\left(t_{k}\right)\right)
\end{array}\right]^{\top}
$$

and

$$
\widetilde{\Upsilon}_{k}=\left[\begin{array}{llll}
y\left(t_{k-w+1}\right) & \ldots & y\left(t_{k-1}\right) & y\left(t_{k}\right)
\end{array}\right]^{\top} .
$$

If $\widetilde{U}_{k}^{\top} \widetilde{U}_{k}$ is full column rank, then we can compute this approximation as

$$
\operatorname{vec}\left(\widetilde{\Gamma}_{k}\right)=\left(\widetilde{U}_{k}^{\top} \widetilde{U}_{k}\right)^{-1} \widetilde{U}_{k}^{\top} \widetilde{\Upsilon}_{k}
$$


It turns out that the property that the elements of $T_{k}^{w}$ can be selected such that $\widetilde{U}_{k}^{\top} \widetilde{U}_{k}$ is full column rank is a property of persistence of excitation which can be characterized in terms of the dimension at $\omega(0)$ of the distribution of

$$
\mathcal{E}=\operatorname{span}\left\{\omega, \omega^{(1)}, \ldots, \omega^{(k)}, \ldots\right\} .
$$

This property is called excitation rank condition, see [17] for a detailed discussion.

To ease the notation we introduce the following definition.

Definition 4. The estimated moment of system (6) at $(s, l)$ is defined as

$$
\widetilde{h \circ \pi}_{N, k}(\omega(t))=\widetilde{\Gamma}_{k} \Omega(\omega(t))
$$

for all $t \in \mathbb{R}$, with $\widetilde{\Gamma}_{k}$ computed using (37).

Then, under technical assumptions, it is possible to show that

$$
\lim _{t \rightarrow \infty}\left(h(\pi(\omega(t)))-\lim _{N \rightarrow M} \widetilde{h \circ \pi}_{N, k}(\omega(t))\right)=0,
$$

i.e. the formula (37) provides an approximation of the moment.

Up to this point we have considered one trajectory $\omega(t)$. While this is sufficient in a linear setting, in which local properties are also global, it may be restrictive in the nonlinear setting. To overcome this limitation, we introduce the socalled “ $\mathfrak{U} / \mathfrak{Y}$ ” variation. This is a modification of the formula (37) operating with multiple trajectories. To this end, it suffices to implement the algorithm replacing the matrices $\widetilde{U}_{k}$ and $\widetilde{\Upsilon}_{k}$ with the matrices

$$
\begin{aligned}
\mathfrak{U} & =\left[\begin{array}{llll}
\tilde{U}_{k}^{1 \top} & \tilde{U}_{k}^{2 \top} & \ldots & \widetilde{U}_{k}^{q \top}
\end{array}\right]^{\top}, \\
\mathfrak{Y} & =\left[\begin{array}{llll}
\widetilde{\Upsilon}_{k}^{1 \top} & \widetilde{\Upsilon}_{k}^{2 \top} & \ldots & \widetilde{\Upsilon}_{k}^{q \top}
\end{array}\right]^{\top},
\end{aligned}
$$

respectively, where $\widetilde{U}_{k}^{i}$ and $\widetilde{\Upsilon}_{k}^{i}$ are the matrices in (35) and (36), respectively, sampled along the trajectory of system (7) starting from the initial condition $\omega(0)=\omega_{0}^{i} \in \mathbb{R}^{\nu}$, with $i=1, \ldots, q, q \geq 1$, where the $\omega_{0}^{i}$ are selected to span a desired set $\bar{W} \subset \mathbb{R}^{\nu}$.

To conclude this part of the tutorial, we have seen that the framework introduced in [29] can be extended to very general classes of systems in a simple manner. More fundamental than the method per se are the ideas behind the connection of moment matching with the concepts of invariance and steady-state. These allow the extension of the framework beyond linear deterministic delay-free differential equations and the derivation of efficient numerical algorithms.

\section{THE LOEWNER FramewORK}

As noted earlier, it has been shown in [11] that the Loewner framework presented in [12] can be viewed as a two-sided moment-matching procedure. In this section of the tutorial we discuss an extension of the Loewner framework presented in [12] for linear systems to affine nonlinear systems. The extension utilizes the interconnectionbased interpretation of the Loewner matrices introduced in
[30]. To accomplish this, Loewner functions are introduced as generalizations of Loewner matrices, which are then used to construct models which can produce the exact same leftand right-Loewner functions, thus achieving interpolation in the Loewner sense. Locally the original model and the interpolating model produce the same response when interconnected with generators derived from the Loewner functions and appropriate initial conditions are chosen.

The method that we discuss has also been utilized to extend the Loewner framework to linear time-varying systems in [31], and to affine nonlinear systems with more general nonlinear generators in [32].

\section{A. Preliminaries}

Classically, the Loewner framework has been defined for descriptor systems (see e.g. [12]). Herein, as in [30], we consider the special case in which the plant is described by equations of the form

$$
\begin{aligned}
& \dot{x}=A x+B u, \\
& y=C x,
\end{aligned}
$$

with state $x(t) \in \mathbb{C}^{n}, u(t) \in \mathbb{C}^{m}, y(t) \in \mathbb{C}^{p}$, and matrices $A$, $B$, and $C$ of appropriate dimensions. For ease of presentation we consider complex-valued signals and matrices. These signals and matrices are obtained via linear coordinate transformations of real valued signals and matrices.

To pose an interpolation problem, and to introduce the Loewner framework, one requires tangential data (see e.g. [33]). Tangential data consist of right tangential data and left tangential data. The right tangential data are written compactly as

$$
\begin{aligned}
& \Lambda=\operatorname{diag}\left[\lambda_{1}, \ldots, \lambda_{\rho}\right] \in \mathbb{C}^{\rho \times \rho}, \\
& R=\left[\begin{array}{lll}
r_{1} & \ldots & r_{\rho}
\end{array}\right] \in \mathbb{C}^{m \times \rho}, \\
& W=\left[\begin{array}{lll}
w_{1} & \ldots & w_{\rho}
\end{array}\right] \in \mathbb{C}^{p \times \rho},
\end{aligned}
$$

and the left tangential data are written compactly as

$$
\begin{aligned}
& M=\operatorname{diag}\left[\mu_{1}, \ldots, \quad \mu_{v}\right] \in \mathbb{C}^{v \times v}, \\
& L=\left[\begin{array}{c}
\ell_{1} \\
\vdots \\
\ell_{v}
\end{array}\right] \in \mathbb{C}^{v \times p}, \quad V=\left[\begin{array}{c}
v_{1} \\
\vdots \\
v_{v}
\end{array}\right] \in \mathbb{C}^{v \times m} .
\end{aligned}
$$

The following assumption is required to guarantee uniqueness of solution to a number of Sylvester equations arising in the Loewner framework.

Assumption 6. The matrices $A, \Lambda$, and $M$ have no common eigenvalues, that is

$$
\sigma(A) \cap \sigma(\Lambda)=\emptyset, \sigma(A) \cap \sigma(M)=\emptyset, \sigma(M) \cap \sigma(\Lambda)=\emptyset .
$$

The goal of the realization problem is to determine a statespace representation of the form (40)-(41) such that the corresponding rational transfer matrix $H(s)=C(s I-A)^{-1} B$ obeys the right interpolation conditions

$$
H\left(\lambda_{i}\right) r_{i}=w_{i}, i=1, \ldots, \rho,
$$


and the left interpolation conditions

$$
\ell_{j} H\left(\mu_{j}\right)=v_{j}, j=1, \ldots, v .
$$

The Loewner matrix, $\mathbb{L}$, and the shifted Loewner matrix, $\sigma \mathbb{L}$, are defined in terms of the tangential data as the unique (by Assumption 6) solution to the Sylvester equations [34]

$$
\mathbb{L} \Lambda-M \mathbb{L}=L W-V R,
$$

and

$$
\sigma \mathbb{L} \Lambda-M \sigma \mathbb{L}=L W \Lambda-M V R .
$$

It is also shown in [12] that $\sigma \mathbb{L}-\mathbb{L} \Lambda=V R$ and that $\sigma \mathbb{L}-M \mathbb{L}=L W$.

The tangential generalized observability matrix, $Y$, and the tangential generalized controllability matrix, $X$, are defined as the unique (by Assumption 6) solution to the equations

$$
Y A+L C=M Y,
$$

and

$$
A X+B R=X \Lambda,
$$

respectively. Having defined the matrices $Y$ and $X$, the matrices $W$ and $V$ can be expressed as

$$
W=C X, \quad V=Y B .
$$

Finally the Loewner matrix and the shifted Loewner matrix can be expressed as

$$
\mathbb{L}=-Y X, \quad \sigma \mathbb{L}=-Y A X .
$$

Remark 6. The matrices $W$ and $V$ contain the moments of the system (40)-(41) at the eigenvalues of $\Lambda$ and $M$ if the following conditions hold: $(\Lambda, R)$ is observable, $(M, L)$ is reachable, $R$ has a right inverse, and $L$ has a left inverse [35].

As shown in [12], if the matrices $\mathbb{L}, \sigma \mathbb{L}, V$, and $W$ associated to the system (40)-(41) are known and $\mathbb{L}$ is nonsingular, then an interpolating system (i.e. a system which matches the tangential data conditions (42) and (43)) with state $r(t) \in \mathbb{C}^{\rho}$, input $u_{r}(t) \in \mathbb{C}^{m}$, and output $y_{r}(t) \in \mathbb{C}^{p}$ is given by

$$
\begin{aligned}
\dot{r}(t) & =\mathbb{L}^{-1} \sigma \mathbb{L} r(t)-\mathbb{L}^{-1} V u_{r}(t), \\
y_{r}(t) & =W r(t) .
\end{aligned}
$$

To allow extending the Loewner framework to more general systems the notion of left-Loewner and right-Loewner matrices has been introduced in [30]. The left-Loewner matrix, $\mathbb{L}^{\ell}$, is defined as the unique (by Assumption ${ }^{5}$ 6) solution to the Sylvester equation

$$
M \mathbb{L}^{\ell}-\mathbb{L}^{\ell} \Lambda=V R,
$$

and the right-Loewner matrix, $\mathbb{L}^{r}$, is defined as the unique solution to the Sylvester equation

$$
\mathbb{L}^{r} \Lambda-M \mathbb{L}^{r}=L W .
$$

\footnotetext{
${ }^{5} \mathrm{We}$ omit this statement for the remainder of this section.
}

It follows trivially that

$$
\mathbb{L}=\mathbb{L}^{\ell}+\mathbb{L}^{r} .
$$

Analogously, the shifted left-Loewner matrix and the shifted right-Loewner matrix, $\sigma \mathbb{L}^{\ell}$ and $\sigma \mathbb{L}^{r}$ respectively, are defined as the unique solution to the Sylvester equations

$$
M \sigma \mathbb{L}^{\ell}-\sigma \mathbb{L}^{\ell} \Lambda=M V R,
$$

and

$$
\sigma \mathbb{L}^{r} \Lambda-M \sigma \mathbb{L}^{r}=L W \Lambda .
$$

Once again, it follows that

$$
\sigma \mathbb{L}=\sigma \mathbb{L}^{\ell}+\sigma \mathbb{L}^{r} .
$$

Moreover, by uniqueness of the solution to (48), (49), (50), and (51) we have that

$$
\sigma \mathbb{L}^{\ell}=M \mathbb{L}^{\ell}, \quad \sigma \mathbb{L}^{r}=\mathbb{L}^{r} \Lambda .
$$

The left- and right-Loewner "objects" yield an interconnection-based interpretation of the Loewner matrices for the system (40)-(41) and its associated tangential data. To show this we introduce two auxiliary systems, defined using the right and the left tangential interpolation data, as ${ }^{6}$

$$
\begin{aligned}
\dot{\zeta}_{r}(t) & =\Lambda \zeta_{r}(t)+\Delta(t), \\
v(t) & =R \zeta_{r}(t),
\end{aligned}
$$

and

$$
\begin{aligned}
\dot{\zeta}_{\ell}(t) & =M \zeta_{\ell}(t)+L \chi(t), \\
\eta(t) & =\zeta_{\ell}(t),
\end{aligned}
$$

with states $\zeta_{r}(t) \in \mathbb{C}^{\rho}$ and $\zeta_{\ell}(t) \in \mathbb{C}^{v}$, inputs $\Delta(t) \in \mathbb{C}^{\rho}$ and $\chi(t) \in \mathbb{C}^{p}$, and outputs $v(t) \in \mathbb{C}^{m}$ and $\eta(t) \in \mathbb{C}^{v}$. We then consider the cascaded system defined by the interconnection equations $u=v$ and $\chi=y$. This system has a state-space realization given by

$$
\begin{aligned}
{\left[\begin{array}{c}
\dot{\zeta}_{r} \\
\dot{x} \\
\dot{\zeta}_{\ell}
\end{array}\right] } & =\left[\begin{array}{ccc}
\Lambda & 0 & 0 \\
B R & A & 0 \\
0 & L C & M
\end{array}\right]\left[\begin{array}{c}
\zeta_{r} \\
x \\
\zeta_{\ell}
\end{array}\right]+\left[\begin{array}{l}
I \\
0 \\
0
\end{array}\right] \Delta, \\
\eta & =\left[\begin{array}{lll}
0 & 0 & I
\end{array}\right]\left[\begin{array}{c}
\zeta_{r} \\
x \\
\zeta_{\ell}
\end{array}\right] .
\end{aligned}
$$

To expose an important property of the Loewner matrices consider the coordinates transformation [30]

$$
\left[\begin{array}{l}
z_{r} \\
z_{c} \\
z_{\ell}
\end{array}\right]=\left[\begin{array}{ccc}
I & 0 & 0 \\
-X & I & 0 \\
\mathbb{L}^{\ell} & Y & I
\end{array}\right]\left[\begin{array}{c}
\zeta_{r} \\
x \\
\zeta_{\ell}
\end{array}\right] .
$$

\footnotetext{
${ }^{6}$ Assuming the matrices $\Lambda$ and $M$ are diagonal is restrictive in practice. It turns out that these matrices do not need to be diagonal when implementing reduced order models. For a discussion of the topic see Appendix A in [32].
} 
The system in the new coordinates is described by the equations

$$
\begin{gathered}
{\left[\begin{array}{c}
\dot{z}_{r} \\
\dot{z}_{c} \\
\dot{z}_{\ell}
\end{array}\right]=\left[\begin{array}{ccc}
\Lambda & 0 & 0 \\
0 & A & 0 \\
0 & 0 & M
\end{array}\right]\left[\begin{array}{c}
z_{r} \\
z_{c} \\
z_{\ell}
\end{array}\right]+\left[\begin{array}{c}
I \\
-X \\
\mathbb{L}^{\ell}
\end{array}\right] \Delta,} \\
\eta=\left[\begin{array}{lll}
\mathbb{L}^{r} & -Y & I
\end{array}\right]\left[\begin{array}{l}
z_{r} \\
z_{c} \\
z_{\ell}
\end{array}\right] .
\end{gathered}
$$

Thus the Loewner matrices can be viewed as the input and output "gains" of three systems connected in parallel such that the impulse response is the same as that of the interconnected system (56)-(57).

We now provide a definition which is crucial for the construction of reduced order models in the Loewner sense.

Definition 5 (Loewner Equivalence). Let $\Sigma$ and $\bar{\Sigma}$ be two systems with left- and right-Loewner matrices $\mathbb{L}^{\ell}, \mathbb{L}^{r}$, and $\overline{\mathbb{L}}^{\ell}$, $\overline{\mathbb{L}}^{r}$, respectively, associated to the matrices $\Lambda, R, M$, and $L$. Then $\Sigma$ and $\bar{\Sigma}$, are called Loewner equivalent at $(\Lambda, R, M, L)$ if $\mathbb{L}^{\ell}=\overline{\mathbb{L}}^{\ell}$ and $\mathbb{L}^{r}=\overline{\mathbb{L}}^{r}$.

The fact that two systems (with transfer functions $H(s)$ and $\bar{H}(s))$ are Loewner equivalent at $(\Lambda, R, M, L)$ is equivalent to both systems satisfying the conditions (42) and (43).

Considering (58)-(59), and assuming that $\Delta$ is bounded and converges to zero, $A$ has only eigenvalues with negative real part, and $\Lambda$ and $M$ have eigenvalues on the imaginary axis, it is easy to see that the response of the system interconnected with the generators is dependent entirely on the generator states and the left- and right-Loewner matrices. Thus, if two exponentially stable systems are Loewner equivalent at $(\Lambda, R, M, L)$ then there exists an initial condition such that the two systems interconnected with the generators have the same response.

We can now formally define what a reduced order model in the Loewner sense is.

Definition 6 (Reduced Order Model). Let $\Sigma$ and $\bar{\Sigma}$ be two systems of order $n$ and $v$, respectively. $\bar{\Sigma}$ is called a reduced order model of $\Sigma$ in the Loewner sense if $\Sigma$ and $\bar{\Sigma}$ are Loewner equivalent at $(\Lambda, R, M, L)$ and $v<n$.

Remark 7. Consider the interconnected system (56)-(57) with associated Loewner matrices $\mathbb{L}^{\ell}, \mathbb{L}^{r}$, and $\mathbb{L}$. Let $\bar{X}$ and $\bar{Y}$ be the tangential generalized controllability and observability matrices, and $\overline{\mathbb{L}}^{\ell}$ and $\overline{\mathbb{L}}^{r}$ be the left- and right-Loewner matrices, for the system given by the equations (46)-(47) interconnected with the generators (52)-(53) and (54)-(55). Then the following is true: $\bar{X}=I, \bar{Y}=-\mathbb{L}, \overline{\mathbb{L}}^{\ell}=\mathbb{L}^{\ell}$, and $\overline{\mathbb{L}}^{r}=\mathbb{L}^{r}$. Therefore given the moments $\bar{W}=W \bar{X}=W$ and $\bar{V}=\bar{Y}\left(-\mathbb{L}^{-1} V\right)=V$ of the reduced order model in the Loewner sense, it is easy to see that the matrices, and therefore the moments of the system (40)-(41), are matched at the eigenvalues of $\Lambda$ and $M$.

We conclude this preliminary section recalling a definition which is instrumental to prove the existence of solution to a number of PDEs arising in the nonlinear framework and which is the nonlinear counterpart of the non-resonance condition in Assumption 6.

Definition 7 ([36, Def. 2]). Given an $n \times n$ matrix $F$, with spectrum $\sigma(F)=\lambda=\left(\lambda_{1}, \ldots, \lambda_{n}\right)$, and constants $C>0$ and $v>0$ we say that a complex number $\mu$ is of type $(C, v)$ with respect to $\sigma(F)$ if for any vector $m=\left(m_{1}, m_{2}, \ldots, m_{n}\right)$ of nonnegative integers we have

$$
|\mu-m \cdot \lambda| \geq \frac{C}{|m|^{v}},
$$

where $|m|=\sum m_{i}>0$.

\section{B. Problem formulation}

In the rest of this section we focus on nonlinear systems described by equations of the form

$$
\begin{aligned}
& \dot{x}(t)=f(x(t))+g(x(t)) u(t), \\
& y(t)=h(x(t)),
\end{aligned}
$$

with state $x(t) \in \mathbb{C}^{n}$, input $u(t) \in \mathbb{C}^{m}$, and output $y(t) \in \mathbb{C}^{p}$, and functions $f: \mathbb{C}^{n} \rightarrow \mathbb{C}^{n}, g: \mathbb{C}^{n} \rightarrow \mathbb{C}^{n \times m}$, and $h: \mathbb{C}^{n} \rightarrow \mathbb{C}^{p}$ of appropriate dimensions, and such that $f(0)=0, g(0)=0$, and $f(\cdot)$ is differentiable. Let $A:=\frac{\partial f}{\partial x}(0)$. For ease of presentation we consider complex valued mappings and signals which are obtained via linear coordinates transformations of real valued mappings and signals. In addition, with some abuse of terminology, we say, for example, that the zero equilibrium of $\dot{x}=f(x)$, with $x(t) \in \mathbb{C}^{n}$ and $f: \mathbb{C}^{n} \rightarrow \mathbb{C}^{n}$, is locally asymptotically stable if the zero equilibrium of the underlying "real" system is locally asymptotically stable.

Assumption 7. The unforced system $\dot{x}=f(x)$ is locally exponentially stable at the origin, that is all eigenvalues of $A$ are in $\mathbb{C}^{-}$.

The goal of this section is to extend the interpolation methods of [12], which has been revisited in Section A, to nonlinear systems of the form (60)-(61) using the state-space interpretation given in [30]. To do this we introduce the notion of Loewner functions which are, in turn, used to introduce the concept of Loewner equivalence at given operating conditions. It is important to note that the following statements regarding the existence of the Loewner functions are local.

\section{Interconnection with linear generators}

To exploit the state-space interpretation of the Loewner matrices given in [30] we begin by constructing two systems. We start with a simple setup given by two systems of the form

$$
\begin{aligned}
\dot{\zeta}_{r}(t) & =\Lambda \zeta_{r}(t)+\Delta(t), \\
v(t) & =R \zeta_{r}(t),
\end{aligned}
$$

and

$$
\begin{aligned}
\dot{\zeta}_{\ell}(t) & =M \zeta_{\ell}(t)+L \chi(t), \\
\eta(t) & =\zeta_{\ell}(t),
\end{aligned}
$$


with states $\zeta_{r}(t) \in \mathbb{C}^{\rho}$ and $\zeta_{\ell}(t) \in \mathbb{C}^{v}$, inputs $\Delta(t) \in \mathbb{C}^{\rho}$ and $\chi(t) \in \mathbb{C}^{p}$, and outputs $v(t) \in \mathbb{C}^{m}$ and $\eta(t) \in \mathbb{C}^{v}$, and with matrices $\Lambda \in \mathbb{C}^{\rho \times \rho}, R \in \mathbb{C}^{m \times \rho}, M \in \mathbb{C}^{v \times v}$, and $L \in \mathbb{C}^{v \times p}$.

The following assumption is imposed to restrict the analysis to bounded signals.

Assumption 8. The matrices $\Lambda$ and $M$ have all eigenvalues on the imaginary axis, and these eigenvalues have geometric multiplicity one.

Consider now the interconnection of the system (60)-(61) with the generators (62)-(63) and (64)-(65), defined via the interconnection equations $u=v$ and $\chi=y$, which yields the state-space representation

$$
\begin{aligned}
{\left[\begin{array}{c}
\dot{\zeta}_{r} \\
\dot{x} \\
\dot{\zeta}_{\ell}
\end{array}\right] } & =\left[\begin{array}{c}
\Lambda \zeta_{r} \\
f(x)+g(x) R \zeta_{r} \\
M \zeta_{\ell}+L h(x)
\end{array}\right]+\left[\begin{array}{l}
I \\
0 \\
0
\end{array}\right] \Delta, \\
\quad \eta & =\zeta_{\ell},
\end{aligned}
$$

with state $\left[\begin{array}{lll}\zeta_{r}^{\top} & x^{\top} & \zeta_{\ell}^{\top}\end{array}\right]^{\top}$, input $\Delta$, and output $\eta$.

\section{Loewner functions}

Before presenting the main results we define the nonlinear enhancements of the tangential generalized controllability and observability matrices and of the Loewner matrices. These are defined in terms of the functions and matrices appearing in the interconnected system (66)-(67). The tangential generalized controllability function, $X: \mathbb{C}^{\rho} \rightarrow \mathbb{C}^{n}$, is defined as the solution, provided it exists, to the PDE with boundary condition

$$
\frac{\partial X}{\partial \zeta_{r}} \Lambda \zeta_{r}=f\left(X\left(\zeta_{r}\right)\right)+g\left(X\left(\zeta_{r}\right)\right) R \zeta_{r}, \quad X(0)=0 .
$$

The following existence conditions are direct consequences of Assumptions 7 and 8 and of the center manifold theory, see [37], and of Lemma 1 in [32].

Proposition 2 (Existence of $X$ ). Consider the PDE (68) with the boundary condition $X(0)=0$. Suppose Assumption 7 and Assumption 8 hold. Then there exists a function $X$ : $\mathbb{C}^{\rho} \rightarrow \mathbb{C}^{n}$ satisfying the partial differential equation (68) with the given boundary condition.

Proposition 3 (Existence of $X$ ). Consider the PDE (68) with the boundary condition $X(0)=0$ and suppose that $f$ is analytic and the map $g$ is constant. Suppose there exist constants $C>0$ and $v>0$ such that all eigenvalues of $A$ are of type $(C, v)$ with respect to $\sigma(\Lambda)$. Then there exists a function $X: \mathbb{C}^{\rho} \rightarrow \mathbb{C}^{n}$ satisfying the partial differential equation (68) with the given boundary condition.

Note that while Proposition 2 implies that $x=X\left(\zeta_{r}\right)$ is a local attractive center manifold, Proposition 3 has no such center manifold implication.

The tangential generalized observability function, $Y$ : $\mathbb{C}^{n} \rightarrow \mathbb{C}^{v}$, is defined as the solution, provided it exists, to the PDE with boundary condition

$$
\frac{\partial Y}{\partial x} f(x)=M Y(x)-\operatorname{Lh}(x), \quad Y(0)=0 .
$$

The following claim holds by Lemma 1 in [32].

Proposition 4 (Existence of $Y$ ). Consider the PDE (69) with the boundary condition $Y(0)=0$ and suppose that $f$ and $h$ are analytic. Suppose there exist constants $C>0$ and $v>0$ such that all eigenvalues of $M$ are of type $(C, v)$ with respect to $\sigma(A)$. Then there exists a function $Y: \mathbb{C}^{n} \rightarrow \mathbb{C}^{v}$ satisfying the partial differential equation (69) with the given boundary condition.

The following corollary is a consequence of Definition 7.

Corollary 1. Suppose that Assumptions 7 and 8 hold. Then the eigenvalues of $A$ are of type $(C, v)$ with respect to $\sigma(\Lambda)$ and the eigenvalues of $M$ are of type $(C, v)$ with respect to $\sigma(A)$.

It follows by Corollary 1 that Proposition 4 holds by Assumptions 7 and 8, and Proposition 3 holds by Assumptions 7 and 8 when $g(\cdot)$ is constant.

Having defined the tangential generalized observability and controllability functions, the nonlinear enhancements of the matrices of moments $V$ and $W$ are given by

$$
V\left(\zeta_{r}\right):=\frac{\partial Y}{\partial x}\left(X\left(\zeta_{r}\right)\right) g\left(X\left(\zeta_{r}\right)\right), \quad W\left(\zeta_{r}\right):=h\left(X\left(\zeta_{r}\right)\right) .
$$

The nonlinear Loewner function is defined in terms of the tangential generalized controllability and observability functions as

$$
\mathbb{L}\left(\zeta_{r}\right):=-Y\left(X\left(\zeta_{r}\right)\right) .
$$

The left-Loewner function, $\mathbb{L}^{\ell}: \mathbb{C}^{\rho} \rightarrow \mathbb{C}^{v}$, is defined as the solution, provided it exists, to the PDE with boundary condition

$$
\frac{\partial \mathbb{L}^{\ell}}{\partial \zeta_{r}} \Lambda \zeta_{r}=M \mathbb{L}^{\ell}\left(\zeta_{r}\right)-V\left(\zeta_{r}\right) R \zeta_{r}, \quad \mathbb{L}^{\ell}(0)=0,
$$

and the right-Loewner function, $\mathbb{L}^{r}: \mathbb{C}^{\rho} \rightarrow \mathbb{C}^{v}$, is defined as

$$
\mathbb{L}^{r}\left(\zeta_{r}\right):=\mathbb{L}\left(\zeta_{r}\right)-\mathbb{L}^{\ell}\left(\zeta_{r}\right)
$$

The next claim follows by a direct application of the main theorem of [36] (see also Lemma 1 in [32]).

Proposition 5 (Existence of $\mathbb{L}^{\ell}$ ). Consider the PDE (70) with the boundary condition $\mathbb{L}^{\ell}(0)=0$ and suppose that $V$ is analytic. Suppose there exist constants $C>0$ and $v>0$ such that all eigenvalues of $M$ are of type $(C, v)$ with respect to $\sigma(\Lambda)$. Then there exists a function $\mathbb{L}^{\ell}: \mathbb{C}^{\rho} \rightarrow \mathbb{C}^{v}$ satisfying the partial differential equation (70) with the given boundary condition.

Remark 8. The preceeding existence conditions utilizing Definition 7 have the advantage that the PDEs (68), (69), and (70) have solutions even for unstable systems. Namely, unstable nonlinear systems can be analyzed in the nonlinear Loewner framework by abstaining from Assumptions 7 and 8. However, when considering unstable systems the relation with the steady-state response is lost.

The definitions introduced thus far show that the Loewner and right-Loewner functions satisfy the PDEs with boundary 
conditions

$$
\frac{\partial \mathbb{L}}{\partial \zeta_{r}} \Lambda \zeta_{r}=M \mathbb{L}\left(\zeta_{r}\right)+L W\left(\zeta_{r}\right)-V\left(\zeta_{r}\right) R \zeta_{r}, \quad \mathbb{L}(0)=0
$$

and

$$
\frac{\partial \mathbb{L}^{r}}{\partial \zeta_{r}} \Lambda \zeta_{r}=M \mathbb{L}^{r}\left(\zeta_{r}\right)+L W\left(\zeta_{r}\right), \quad \mathbb{L}^{r}(0)=0 .
$$

The shifted Loewner function, $\sigma \mathbb{L}: \mathbb{C}^{\rho} \rightarrow \mathbb{C}^{v}$, is defined in terms of the left- and right-Loewner functions as

$$
\sigma \mathbb{L}\left(\zeta_{r}\right):=M \mathbb{L}^{\ell}\left(\zeta_{r}\right)+\frac{\partial \mathbb{L}^{r}}{\partial \zeta_{r}} \Lambda \zeta_{r}
$$

which implies that

$$
\sigma \mathbb{L}\left(\zeta_{r}\right)=M \mathbb{L}\left(\zeta_{r}\right)+L W\left(\zeta_{r}\right)=\frac{\partial \mathbb{L}}{\partial \zeta_{r}} \Lambda \zeta_{r}+V\left(\zeta_{r}\right) R \zeta_{r}
$$

and

$$
\sigma \mathbb{L}\left(\zeta_{r}\right)=-\frac{\partial Y}{\partial x}\left(X\left(\zeta_{r}\right)\right) f\left(X\left(\zeta_{r}\right)\right)
$$

Remark 9. If the system (60)-(61) is linear then the solution to the PDEs (68), (69), and (70) becomes $X\left(\zeta_{r}\right)=X \zeta_{r}$, $Y(x)=Y x$, and $\mathbb{L}^{\ell}\left(\zeta_{r}\right)=\mathbb{L}^{\ell} \zeta_{r}$, where $X, Y$, and $\mathbb{L}^{\ell}$ are the solutions to the Sylvester equations (44), (45), and (48). Thus the linear Loewner objects are recovered.

\section{E. Loewner coordinates}

To expose the relation between the Loewner functions and the interconnection of systems (66)-(67) we select a specific set of coordinates.

Theorem 6. Consider the system (66)-(67). The coordinates transformation

$$
\left[\begin{array}{c}
z_{r} \\
z_{c} \\
z_{\ell}
\end{array}\right]:=\left[\begin{array}{c}
\zeta_{r} \\
x-X\left(\zeta_{r}\right) \\
\zeta_{\ell}+Y(x)+\mathbb{L}^{\ell}\left(\zeta_{r}\right)
\end{array}\right]
$$

is such that the system in the new coordinates is described by the equations

$$
\begin{aligned}
& {\left[\begin{array}{c}
\dot{z}_{r} \\
\dot{z}_{c} \\
\dot{z}_{\ell}
\end{array}\right]=} {\left[\begin{array}{ccc}
\Lambda & 0 & 0 \\
0 & \widetilde{A}\left(z_{c}+X\left(z_{r}\right), z_{r}\right) & 0 \\
0 & \widetilde{G}\left(z_{c}+X\left(z_{r}\right), z_{r}\right) & M
\end{array}\right]\left[\begin{array}{c}
z_{r} \\
z_{c} \\
z_{\ell}
\end{array}\right] } \\
&+ {\left[\begin{array}{c}
I \\
-\frac{\partial X}{\partial \zeta_{r}}\left(z_{r}\right) \\
\frac{\partial \mathbb{L}^{\ell}}{\partial \zeta_{r}}\left(z_{r}\right)
\end{array}\right] \Delta, } \\
& \eta=\mathbb{L}^{r}\left(z_{r}\right)-\tilde{Y}\left(z_{c}+X\left(z_{r}\right)\right) z_{c}+z_{\ell}
\end{aligned}
$$

where $z_{r}(t) \in \mathbb{C}^{\rho}, z_{c}(t) \in \mathbb{C}^{n}, z_{\ell}(t) \in \mathbb{C}^{v}$, and where $\widetilde{A}: \mathbb{C}^{n} \times \mathbb{C}^{\rho} \rightarrow \mathbb{C}^{n \times n}, \widetilde{G}: \mathbb{C}^{n} \times \mathbb{C}^{\rho} \rightarrow \mathbb{C}^{v \times n}$, and $\widetilde{Y}:$ $\mathbb{C}^{n} \rightarrow \mathbb{C}^{v \times n}$.

Note that, by Assumption 7, for any sufficiently small $x(0)$ and $\zeta_{r}(0)$, the solutions of the interconnected systems approach the center manifold $x=X\left(\zeta_{r}\right)$ exponentially fast, hence $z_{c}$ approaches zero provided $\Delta$ is sufficiently small and converges to zero, and the system has a converging input converging state property. On the center manifold, that is for $x=X\left(\zeta_{r}\right)$, or $z_{c}=0$, one has

$$
\begin{aligned}
& \dot{z}_{r}=\Lambda z_{r}+\Delta \\
& \dot{z}_{\ell}=M z_{\ell}+\frac{\partial \mathbb{L}^{\ell}}{\partial \zeta_{r}}\left(z_{r}\right) \Delta
\end{aligned}
$$

and

$$
\eta=\mathbb{L}^{r}\left(z_{r}\right)+z_{\ell}
$$

that is the system restricted to the center manifold contains only information on the Loewner functions.

\section{F. Loewner equivalent model}

In this section the concept of reduced order model in the Loewner sense for nonlinear systems is introduced. In addition, a nonlinear system, reminiscent of the linear system (46)-(47), which interpolates the Loewner functions defined by the PDEs (68), (69), and (70), is constructed. Given that the frequency domain interpretations of (42) and (43) hold little meaning in the nonlinear context, we start by describing what we mean by an interpolant when referring to nonlinear systems.

Definition 8 (Loewner Equivalence). Let $\Sigma$ and $\bar{\Sigma}$ be two systems described by equations of the form (60)-(61) admitting left- and right-Loewner functions $\mathbb{L}^{\ell}(\cdot), \mathbb{L}^{r}(\cdot)$, and $\overline{\mathbb{L}}^{\ell}(\cdot), \overline{\mathbb{L}}^{r}(\cdot)$, respectively, associated to the matrices $\Lambda, R$, $M$, and $L$. Then $\Sigma$ and $\bar{\Sigma}$ are called Loewner equivalent at $(\Lambda, R, M, L)$ if $\mathbb{L}^{\ell}\left(\zeta_{r}\right)=\overline{\mathbb{L}}^{\ell}\left(\zeta_{r}\right)$ and $\mathbb{L}^{r}\left(\zeta_{r}\right)=\overline{\mathbb{L}}^{r}\left(\zeta_{r}\right)$ in a neighbourhood of the origin.

Consistently, we say that a nonlinear system interpolates another nonlinear system (in the Loewner sense) at $(\Lambda, R, M, L)$ if the two systems are Loewner equivalent at $(\Lambda, R, M, L)$. That is, for the same matrices $\Lambda, R, M, L$, the interpolating system possesses the exact same left- and right-Loewner functions.

The property of Loewner equivalence has a strong implication on the steady-state behavior of the system. By Theorem 6, recalling Assumptions 7 and 8, assuming the foregoing stability conditions hold, $\Delta$ is sufficiently small, bounded, and converges to zero, and the plant state $x$ has not left the region of attraction of the origin (i.e. $x$ still approaches the center manifold $X\left(\zeta_{r}\right)$ ), it is easy to see that the response of the system interconnected with the generators is dependent entirely on the generator states and the left- and right-Loewner functions. Thus, if two locally exponentially stable systems are Loewner equivalent at $(\Lambda, R, M, L)$ then there exist initial conditions corresponding to points on the manifold $x=X\left(\zeta_{r}\right)$ such that the two systems interconnected with the generators have the same response.

We can now define what a reduced order model is in the Loewner sense.

Definition 9 (Reduced Order Model). Let $\Sigma$ and $\bar{\Sigma}$ be two systems of order $n$ and $v$, respectively. $\bar{\Sigma}$ is called a reduced order model of $\Sigma$ in the Loewner sense if $\Sigma$ and $\bar{\Sigma}$ are Loewner equivalent at $(\Lambda, R, M, L)$ and $v<n$. 
We now construct a nonlinear system which is Loewner equivalent at $(\Lambda, R, M, L)$ to (66)-(67), given that the Loewner functions of (66)-(67) are known.

Theorem 7. Consider the interconnected system (66)-(67) with $\rho=v$. Let $\mathbb{L}^{\ell}(\cdot), \mathbb{L}^{r}(\cdot), \mathbb{L}(\cdot), \sigma \mathbb{L}(\cdot), V(\cdot)$, and $W(\cdot)$ be the associated Loewner functions. Assume that $\frac{\partial \mathbb{L}}{\partial \zeta_{r}}$ is non-singular. Define the system

$$
\begin{aligned}
\frac{\partial \mathbb{L}}{\partial \zeta_{r}}(r) \dot{r} & =\sigma \mathbb{L}(r)-V(r) u_{r}, \\
y_{r} & =W(r),
\end{aligned}
$$

with state $r(t) \in \mathbb{C}^{\rho}$, input $u_{r}(t) \in \mathbb{C}^{m}$, and output $y_{r}(t) \in \mathbb{C}^{p}$. Then the system (71)-(72) is Loewner equivalent at $(\Lambda, R, M, L)$ to the system (60)-(61).

Remark 10. Similarly to Remark 7, consider the interconnected system (66)-(67) with associated Loewner functions $\mathbb{L}^{\ell}(\cdot), \mathbb{L}^{r}(\cdot)$, and $\mathbb{L}(\cdot)$. Let $\bar{X}(\cdot)$ and $\bar{Y}(\cdot)$ be the tangential generalized controllability and observability functions, and $\overline{\mathbb{L}}^{\ell}(\cdot)$ and $\overline{\mathbb{L}}^{r}(\cdot)$ be the left- and right-Loewner functions, for the system given by the equations (71)-(72) interconnected with the generators (62)-(63) and (64)-(65). Then $\bar{X}\left(\zeta_{r}\right)=\zeta_{r}$, $\bar{Y}(r)=-\mathbb{L}(r), \overline{\mathbb{L}}^{\ell}\left(\zeta_{r}\right)=\mathbb{L}^{\ell}\left(\zeta_{r}\right)$, and $\overline{\mathbb{L}}^{r}\left(\zeta_{r}\right)=\mathbb{L}^{r}\left(\zeta_{r}\right)$. Therefore given the moments

$$
\bar{W}\left(\zeta_{r}\right)=W\left(\bar{X}\left(\zeta_{r}\right)\right)=W\left(\zeta_{r}\right)
$$

and

$\bar{V}\left(\zeta_{r}\right)=-\frac{\partial \bar{Y}}{\partial r}\left(\bar{X}\left(\zeta_{r}\right)\right)\left(\frac{\partial \mathbb{L}}{\partial \zeta_{r}}\left(\bar{X}\left(\zeta_{r}\right)\right)\right)^{-1} V\left(\bar{X}\left(\zeta_{r}\right)\right)=V\left(\zeta_{r}\right)$

of the reduced order model in the Loewner sense, it is easy to see that the Loewner functions, and therefore the moments of system (60)-(61), are matched at $(\Lambda, R, M, L)$.

We conclude this section noting that we have presented a new method for the model reduction of nonlinear systems. This method extends an interconnection-based interpretation of the Loewner framework developed in [30]. In addition to matching the Loewner functions, the reduced order model in the Loewner sense matches the moments of the original model. Note, finally, that the interconnection-based interpretation has also been utilized to extend the Loewner framework to linear time-varying systems in [31], and to affine nonlinear systems with nonlinear generators in [32].

\section{Applications to Optimal Control and Wave ENERGY CONVERSION}

This part of the tutorial shows that, besides being a powerful tool for model reduction purposes, the parameterisation of the steady-state response of a system in terms of moments can be useful to approximate optimal control problems (OCPs). In particular, we illustrate this claim by solving the WEC energymaximising OCP using a moment-based representation, where the moment-based parameterisation is particularly well matched to the application, where steady-state energymaximisation is of paramount importance, resulting in an efficient control implementation.
Before discussing wave energy systems, and their associated OCP, the following definition is introduced.

Definition 10. Let $\Sigma$ be a SISO (stable) LTI system described in state-space by the set of matrices $(A, B, C)$, with output $y$. We also call the matrix $\underline{Y}=C \Pi$, with $\Pi$ solution of (3), the moment-domain equivalent of $y$.

\section{A. Wave energy systems}

We begin this section by recalling that the equation of motion for a controlled WEC, under the assumptions of linear potential flow theory (see, for instance, [38]), can be expressed in terms of Cummins' equation [39], i.e. ${ }^{7}$

$$
m \ddot{x}=f_{r}+f_{r e}+f_{e}-u,
$$

where $m$ is the mass of the device, $x: \mathbb{R}_{\geq 0} \rightarrow \mathbb{R}$ the device excursion (displacement), $f_{e}: \mathbb{R}_{>0} \rightarrow \mathbb{R}$, the wave excitation force (uncontrollable input), $f_{r e}$ the hydrostatic restoring force, $f_{r}$ the radiation force, and $u$ the exerted control (PTO) force. The linearised hydrostatic force can be written as $f_{r e}(t)=$ $-s_{h} x(t)$, where $s_{h}=\rho g D$ denotes the hydrostatic stiffness, with $\rho$ the water density, $D$ the characteristic area of the device, and $g$ the gravitational constant. The radiation force $f_{r e}$ is modelled based on linear potential theory and, using the well-known Cummins' equation [39], can be written as

$$
f_{r}(t)=-\mu_{\infty} \ddot{x}(t)-\int_{\mathbb{R}_{\geq 0}} k_{r}(\tau) \dot{x}(t-\tau) d \tau
$$

where $\mu_{\infty}=\lim _{\omega \rightarrow+\infty} \tilde{A}(\omega)>0$ is the so-called addedmass at infinite frequency, $\tilde{A}(\omega)$ is the radiation added mass 8 and $k_{r}: \mathbb{R}_{\geq 0} \rightarrow \mathbb{R}$ is the (causal) radiation impulse response function containing the memory effect of the fluid response. Finally, the equation of motion of the WEC is given by

$$
\Sigma:\left\{\begin{array}{l}
\ddot{x}=\mathcal{M}^{-1}\left(-k_{r} * \dot{x}-s_{h} z+f_{e}-u\right), \\
y=\dot{x}=v
\end{array}\right.
$$

with $\mathcal{M}=m+\mu_{\infty}$, and $y=\dot{x}=v$ is the output of $\Sigma$ (assuming the velocity as the measurable output of the device). We note that equation (75) is of a Volterra integrodifferential form, specifically of the convolution class (see [40]).

Remark 11. The internal stability of (75) is guaranteed, in the Lyapunov sense, for any physically meaningful parameters and impulse response mapping $k_{r}$ involved (see [38]).

\section{B. Moment-based representation of a WEC}

We begin by rewriting the equation of motion (75) in a more suitable structure for the upcoming discussion, as

$$
\Sigma:\left\{\dot{w}=A w+B\left(-k_{r} * C w+f_{e}-u\right), \quad y=C w,\right.
$$

for $t \in \mathbb{R}_{\geq 0}$, where $w(t)=\left[\begin{array}{ll}x(t) & \dot{x}(t)\end{array}\right]^{\top} \in \mathbb{R}^{2}$ contains displacement and velocity for the (single) degree-of-freedom

\footnotetext{
${ }^{7}$ In what follows the dependence on $t$ is dropped whenever convenient. ${ }^{8}$ See [38] for the definition of $\tilde{A}(\omega)$.
} 
involved in the equation of motion, and the (constant) matrices $A \in \mathbb{R}^{2 \times 2}, B \in \mathbb{R}^{2}$ and $C^{\top} \in \mathbb{R}^{2}$ are defined as

$$
A=\left[\begin{array}{cc}
0 & 1 \\
-\mathcal{M} s_{h} & 0
\end{array}\right], \quad B=\left[\begin{array}{c}
0 \\
\mathcal{M}^{-1}
\end{array}\right], \quad C=\left[\begin{array}{l}
0 \\
1
\end{array}\right]^{\top} .
$$

Following the moment-based theory presented in Section II, the mappings corresponding to both external inputs, i.e. the wave excitation $f_{e}$, and control force $u$, are written in terms of an autonomous single-output signal generator, i.e.

$$
\dot{\omega}=S \omega, \quad f_{e}=L_{e} \omega, \quad u=L_{u} \omega
$$

for $t \in \mathbb{R}_{\geq 0}$, with $\omega(t) \in \mathbb{R}^{\nu}, S \in \mathbb{R}^{\nu \times \nu}$ and $\left\{L_{e}^{\top}, L_{u}^{\top}\right\} \in \mathbb{R}^{\nu}$.

The standard assumption for the mathematical representation of wave excitation forces in ocean engineering applications is that $f_{e}$ can be written as a finite sum of harmonics of a so-called fundamental frequency $f_{0}$ [41]. In the light of this, the dynamic matrix $S$ in equation (78) is defined in block-diagonal form as

$$
S=\bigoplus_{p=1}^{\tilde{f}}\left[\begin{array}{cc}
0 & p f_{0} \\
-p f_{0} & 0
\end{array}\right]
$$

where $\nu=2 \tilde{f}$, and hence $\sigma(S) \subset \mathbb{C}_{0}$.

Given that the input to the WEC system is composed of a (linear) combination of both the wave excitation force $f_{e}$ and the control law $u$, the following assumption is required to have a well-posed definition of moments (i.e. analogous to Assumption 3).

Assumption 9. The triple of matrices $\left(L_{e}-L_{u}, S, \omega(0)\right)$ is minimal, i.e. observable and excitable.

Remark 12. Note that the previous assumption is without loss of generality as the signal generator is user-defined and so it can always be constructed such that the assumption holds.

The following 'step' is to compute the parameterisation of the steady-state output response of system (76) in terms of the corresponding moments, i.e.

$$
v_{\mathrm{ss}}(t)=\underline{V} \omega(t)
$$

where $\underline{V}$ is the moment-domain equivalent of the output $v$ of the WEC system. Such a representation is explicitly used to solve the WEC optimal problem in Section V-C. Nonetheless, a direct application of Lemma 1 is not possible, given the presence of the non-parametric convolution operator in (76). This issue is briefly discussed in the following.

Adopting similar theoretical arguments to those in Lemma 1 , the moment of the WEC system $\Sigma$ at the signal generator $\left(S, L_{e}-L_{u}\right)$ can be computed by solving a specific invariance equation, which can be written, for the WEC case, as

$$
A \Pi+B\left(L_{e}-L_{u}-\underline{K}_{r}\right)=\Pi S,
$$

where $\Pi \in \mathbb{R}^{2 \times \nu}$ and $\underline{K}_{r}^{\top} \in \mathbb{R}^{\nu}$ is the moment-domain equivalent of the radiation convolution term.
Remark 13. If Assumption 9 holds, then the moment-domain equivalent of $v$ can be directly expressed as $\underline{V}=C \Pi$.

The term $\underline{K}_{r}$ clearly depends ${ }^{9}$ on $\Pi$, hence one cannot yet solve (81), until it is properly defined. In the following, we recall the definition of $\underline{K}_{r}$ from [10], to later solve (81).

Lemma 2. [10] The moment-domain equivalent of the convolution integral in (76) can be computed as

$$
\underline{K}_{r}=\underline{V} \mathscr{R}
$$

where the operator $\mathscr{R} \in \mathbb{R}^{\nu \times \nu}$ is a block-diagonal matrix ${ }^{10}$ defined in terms of the Fourier transform of the impulse response mapping $k_{r}$, evaluated at the set $\sigma(S)$.

Remark 14. As explicitly expressed in Lemma 2, this moment-based strategy does not require an a-priori parametric approximation of the radiation force (convolution) term, but actually provides an analytical description of the convolution operation in moment-domain.

Lemma 3. [10] Suppose Assumption 9 holds. Then, the moment-domain equivalent of the output $y$ of system (76) (the velocity of the device $v$ ) can be uniquely determined as

$$
\underline{V}=\left(L_{e}-L_{u}\right) \Phi_{\mathscr{R}}^{\top},
$$

where the matrix $\Phi_{\mathscr{R}} \in \mathbb{R}^{\nu \times \nu}$ is defined as

$$
\begin{aligned}
\Phi_{\mathscr{R}} & =\left(I_{\nu} \otimes C\right) \Phi^{-1}\left(I_{\nu} \otimes-B\right), \\
\Phi & =S \otimes I_{2}+I_{\nu} \otimes A+\mathscr{R}^{\boldsymbol{\top}} \otimes-B C .
\end{aligned}
$$

\section{A linear energy-maximising control solution}

The WEC optimal control design entails an energymaximisation criterion, where the objective is to maximise the absorbed energy from ocean waves over a finite time interval $\mathcal{T}=[0, T] \subset \mathbb{R}_{\geq 0}$. This energy-maximising control procedure can be cast as an optimal control problem, with objective function $\mathcal{J}$, defined as

$$
\mathcal{J}(u)=\frac{1}{T} \int_{\mathcal{T}} u(\tau) v(\tau) d \tau
$$

where $u: \mathcal{T} \rightarrow \mathbb{R}$ denotes the control (PTO) force. In addition, constraints on both the displacement and velocity of the WEC, $x$ and $v$, and the exerted control force $u$, are considered, as

$$
\mathscr{C}:\left\{|x(t)| \leq X_{\max },|v(t)| \leq V_{\max },|u(t)| \leq U_{\max },\right.
$$

with $t \in \mathcal{T}$, and where $\left\{X_{\max }, V_{\max }, U_{\max }\right\} \subset \mathbb{R}^{+}$.

Given the control objective function defined in (85), the governing dynamics of the WEC in (76), and the set of state and input constraints defined in (86), the constrained energy-maximising OCP can be posed as follows.

\footnotetext{
${ }^{9}$ Note that the convolution operator depends on the velocity of the WEC

${ }^{10}$ The specific expression of $\mathscr{R}$ can be found in [10].
} 
Problem 1 (Energy-maximising OCP). Find an optimal control input $u^{\text {opt }}: \mathcal{T} \rightarrow \mathbb{R}$ such that

$$
\begin{aligned}
u^{\mathrm{opt}} & =\arg \max _{u} \mathcal{J}(u), \\
& \text { subject to: } \\
& \left\{\begin{array}{l}
\text { WEC dynamics } \Sigma(76), \\
\text { state and input constraints } \mathscr{C}(86) .
\end{array}\right.
\end{aligned}
$$

The results presented in Section V-B can be used to approximate the energy-maximising OCP of Problem 1, making explicit use of the connection between moments and the steady-state behaviour of system (76). In the following, we provide a definition of the so-called moment-based energymaximising $O C P$. Note that we do not yet include the set of state and input constraints defined in (86). These are explicitly incorporated in Section V-C.1.

Problem 2 (Moment-based energy-maximising OCP). Suppose Assumption 9 holds. Find the optimal control input $\tilde{u}^{\text {opt }}=L_{u}^{\mathrm{opt}} \omega$ such that

$$
\begin{aligned}
& L_{u}^{\mathrm{opt}}=\arg \max _{L_{u}^{\top} \in \mathbb{R}^{\nu}} \frac{1}{T} \int_{\mathcal{T}} L_{u} \omega(\tau) \underline{V} \omega(\tau) d \tau, \\
& \quad \text { subject to: } \\
& \quad \underline{V}-\left(L_{e}-L_{u}\right) \Phi_{\mathscr{R}}^{\top}=0,
\end{aligned}
$$

The main idea behind Problem 2 relies on substituting the integro-differential (equality) constraint, corresponding with the WEC dynamics (76), by the algebraic equation (83). In other words, the moment-based OCP posed in Problem 2 explicitly utilises the steady-state (output) behaviour of system (76), parameterised in terms of $\underline{V}$, i.e. $v_{\mathrm{ss}}=\underline{V} \omega(t)$ (see equation (80)), to solve for the corresponding optimal control input $\tilde{u}^{\text {opt }}$, in terms of the signal generator (78). The main advantage of Problem 2, as opposed to Problem 1, is that it can be solved in terms of a tractable finite-dimensional quadratic program $(\mathrm{QP})$, as recalled in the following.

Lemma 4. [10] Suppose Assumption 9 holds. Then, the solution of the (motion unconstrained) moment-based energymaximising OCP, posed in Problem 2, can be computed as $\tilde{u}^{\mathrm{opt}}=L_{u}^{\mathrm{opt}} \omega$, where $L_{u}^{\mathrm{opt}}$ is the solution of the QP problem

$$
L_{u}^{\mathrm{opt}}=\arg \max _{L_{u}^{\top} \in \mathbb{R}^{\nu}}-\frac{1}{2} L_{u} \Phi_{\mathscr{R}}^{\top} L_{u}^{\top}+\frac{1}{2} L_{e} \Phi_{\mathscr{R}}^{\top} L_{u}^{\top} .
$$

Remark 15. As shown in [10], the moment-based QP problem (89) is strictly concave for the WEC case, hence systematically guaranteeing a unique global maximiser.

1) Handling of state and input constraints: Using the moment-based representations developed throughout Section V-B, the set of state and input constraints (86) can be mapped using their respective moment-domain equivalents ${ }^{11}$, as

$$
\mathscr{C} \mapsto\left\{\begin{array}{l}
\left|\underline{V} S^{-1} \omega(t)\right| \leq X_{\max }, \\
|\underline{V} \omega(t)| \leq V_{\max }, \\
\left|L_{u} \omega(t)\right| \leq U_{\max } .
\end{array}\right.
$$

\footnotetext{
${ }^{11}$ Note that the moment-domain equivalent of the displacement $x$ can be expressed as $\underline{V} S^{-1}$, following [9].
}

Let $\mathcal{T}_{c} \subset \mathcal{T}$, be a finite set of (specified) uniformly-spaced time instants. The constraints defined in (90) can be enforced at the set $\mathcal{T}_{c}$, i.e. using a collocation approach. This gives origin to a set of linear inequality constraints in $L_{u}$, i.e.

$$
L_{u} \mathcal{A}_{x} \leq \mathcal{B}_{x}, \quad L_{u} \mathcal{A}_{v} \leq \mathcal{B}_{v}, \quad L_{u} \mathcal{A}_{u} \leq \mathcal{B}_{u}
$$

which can be directly incorporated to the QP problem of Lemma X. We omit the explicit definition of the pairs of matrices $\left(\mathcal{A}_{x}, \mathcal{B}_{x}\right),\left(\mathcal{A}_{v}, \mathcal{B}_{v}\right)$ and $\left(\mathcal{A}_{u}, \mathcal{B}_{u}\right)$, which corresponds with displacement, velocity and control input, respectively, for economy of space. These can be found in [10], [42].

2) Case study: To demonstrate the performance of the moment-based energy-maximising control of Section V-C, the CorPower-like wave energy device, presented in Figure 1 , is considered, and constrained to oscillate in heave. SWL denotes the still water level and indicates the equilibrium position of the device. The set of state and input constraints in (86) is such that, $Z_{\max }=2[\mathrm{~m}], V_{\max }=2[\mathrm{~m} / \mathrm{s}]$, and $U_{\max }=1 \times 10^{6}[\mathrm{~N}]$.

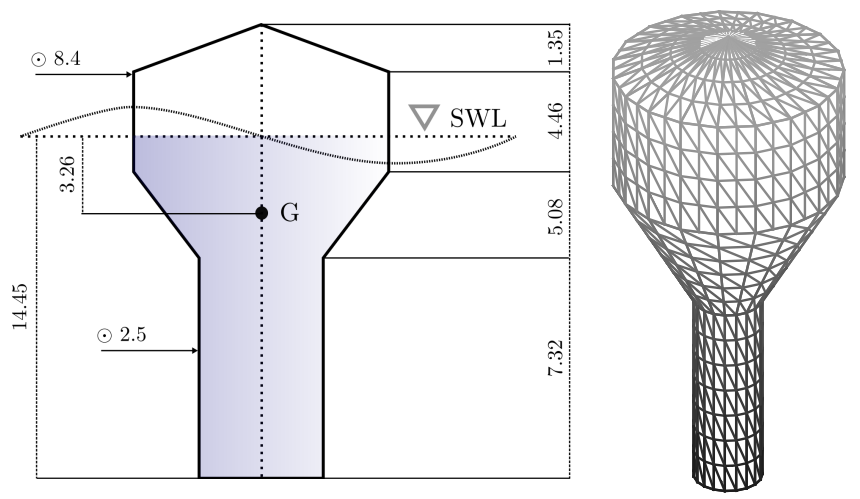

Fig. 1. Full-scale CorPower-like device. The acronym SWL stands for still water level and the letter $\mathbf{G}$ is used to denote the center of gravity.

We consider input waves generated stochastically from a JONSWAP spectral density function [43] with a fixed significant wave height $\bar{H}_{w}$ of 2 [m], varying peak period $\bar{T}_{w} \in[5,12]$ [s] and peak enhancement factor $\gamma=3.3$. The total time-length (fundamental period) of each wave record is set to of $T=120$ [s].

Figure 2 presents performance results, in terms of energy absorption, under both displacement and velocity constraints, for both the moment-based approach presented in this tutorial, and a state-of-the-art impedance-matching-based controller (data extracted from [44]). Note that the latter family of controllers is indeed the most frequently-used approach for this energy-maximising application. As discussed in [44], not only this moment-based approach outperforms wellestablished strategies in the field in terms of energy-absorption (as can be appreciated in Figure 2), but it can be efficiently solved in real-time, given the nature of the objective function when using a moments.

Finally, Figure 3 illustrates the (steady-state) WEC motion (a) under optimally controlled conditions, along with the corresponding moment-based energy-maximising control law (b), for a particular sea state, characterised by a JONSWAP 


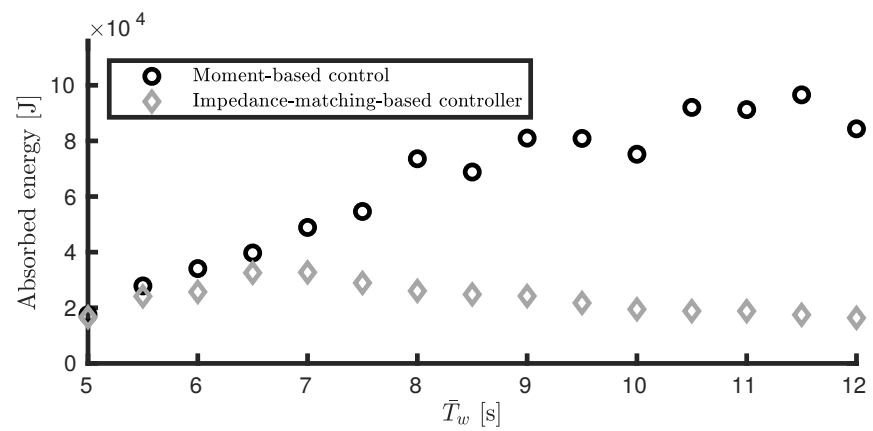

Fig. 2. Constrained (displacement and velocity) energy absorption for the moment-based energy-maximising controller and a state-of-the-art impedancematching-based controller (data extracted from [44])

spectrum with $\bar{H}_{w}=2[\mathrm{~m}]$ and $\bar{T}_{w}=8[\mathrm{~s}]$. As can be appreciated from Figure 3, the moment-based strategy is able to maximise energy absorption while systematically respecting both state and input constraints, according to the control design objective.

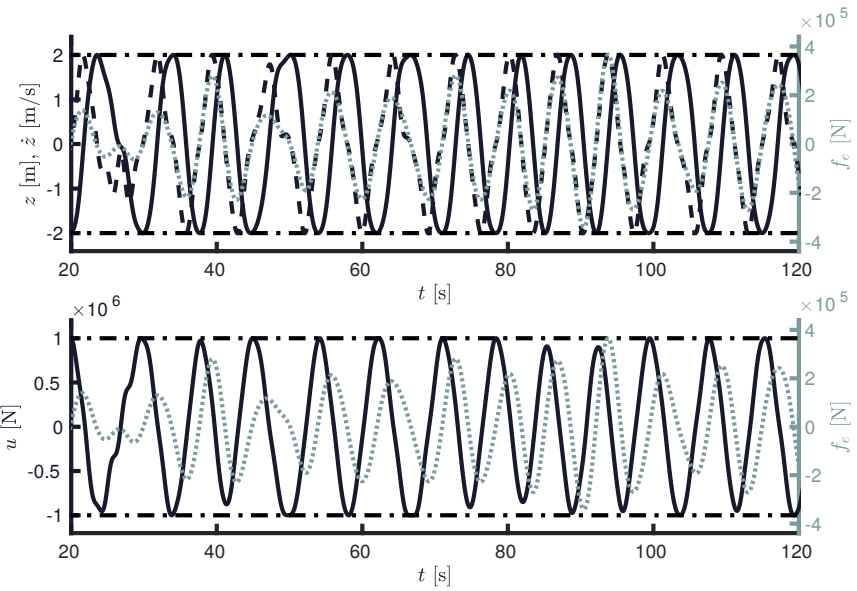

Fig. 3. Motion results for irregular wave excitation. (a) shows displacement (left axis, solid), velocity (left axis, dashed) and wave excitation force input (right axis, dotted), for the WEC system. (b) presents the moment-based control input (left axis, solid), used to elicit the corresponding motion results, along with the wave excitation force input (right axis, dotted). The horizontal dash-dotted lines represent constraint limits.

\section{Towards nonlinear and robust control}

While the system model in (75) and the control solution presented in Section V-C focus on linear dynamics, a main attraction of moment-based representation and control is the relatively straightforward extension to the nonlinear case, following the development in Section II. Indeed, particularly under controlled conditions, which tend to exaggerate device motion [45], hydrodynamic nonlinearities become active and need to be addressed.

To this end, progress has been made in the application of nonlinear moment-based control to wave energy systems [46], demonstrating that a convex nonlinear program can be attained, under mild assumptions about the nature of the nonlinearity. Model nonlinearity could also potentially be addressed using a robust control, based around a nominal linear model, and progress on this has been reported in [47]. However, both the nonlinear and robust wave energy control applications are beyond the scope of this tutorial paper.

\section{Conclusions}

This tutorial paper has presented an overview of the stateof-the art on model reduction by moment matching for nonlinear systems. The fundamental notion of moment for nonlinear systems has been recalled and this has been used to define moments for a wide class of dynamical systems. It has also been shown that this notion is the stepping stone for developing reduced order models both analytically and from measured data. In addition, we have revisited the linear theory; we have discussed recent results on model reduction for hybrid and stochastic systems; we have presented the Loewner framework for nonlinear model reduction; and we have discussed how these ideas and tools can be exploited in optimal control problems, with special attention to problems arising in wave energy conversion.

\section{REFERENCES}

[1] A. Antoulas, Approximation of Large-Scale Dynamical Systems. Philadelphia, PA: SIAM Advances in Design and Control, 2005.

[2] G. Scarciotti and A. Astolfi, "Nonlinear model reduction by moment matching," Foundations and Trends in Systems and Control, vol. 4, no. 3-4, pp. 224-409, 2017.

[3] K. Gallivan, A. Vandendorpe, and P. Van Dooren, "Sylvester equations and projection-based model reduction," Journal of Computational and Applied Mathematics, vol. 162, no. 1, pp. 213-229, 2004.

[4] K. A. Gallivan, A. Vandendorpe, and P. Van Dooren, "Model reduction and the solution of Sylvester equations," in 17th International Symposium on Mathematical Theory of Networks and Systems, Kyoto, Japan, 2006.

[5] A. Astolfi, "Model reduction by moment matching," IFAC Proceedings Volumes, vol. 40, no. 12, pp. 577 - 584, 2007, 7th IFAC Symposium on Nonlinear Control Systems.

[6] _ - "A new look at model reduction by moment matching for linear systems," in Proceedings of the 46th IEEE Conference on Decision and Control, Dec 2007, pp. 4361-4366.

[7] - "Model reduction by moment matching for nonlinear systems," Proceedings of the 47th IEEE Conference on Decision and Control, pp. 4873-4878, 2008.

[8] _ - "Model reduction by moment matching for linear and nonlinear systems," IEEE Transactions on Automatic Control, vol. 55, no. 10, pp. 2321-2336, 2010.

[9] G. Scarciotti and A. Astolfi, "Moment-based discontinuous phasor transform and its application to the steady-state analysis of inverters and wireless power transfer systems," IEEE Transactions on Power Electronics, vol. 31, no. 12, pp. 8448-8460, 2016.

[10] N. Faedo, G. Scarciotti, A. Astolfi, and J. V. Ringwood, "Energymaximising control of wave energy converters using a moment-domain representation," Control Engineering Practice, vol. 81, pp. 85 - 96, 2018.

[11] P. Schulze, T. C. Ionescu, and J. M. Scherpen, "Families of moment matching-based reduced order models for linear descriptor systems," in 2016 European Control Conference, 2016, pp. 1964-1969.

[12] A. Mayo and A. Antoulas, "A framework for the solution of the generalized realization problem," Linear algebra and its applications, vol. 425, no. 2-3, pp. 634-662, 2007.

[13] A. C. Antoulas, I. V. Gosea, and A. C. Ionita, "Model reduction of bilinear systems in the Loewner framework," SIAM Journal on Scientific Computing, vol. 38, no. 5, pp. B889-B916, 2016.

[14] I. V. Gosea, M. Petreczky, and A. C. Antoulas, "Data-driven model order reduction of linear switched systems in the Loewner framework," SIAM Journal on Scientific Computing, vol. 40, no. 2, pp. B572-B610, 2018.

[15] I. V. Gosea and A. C. Antoulas, "Data-driven model order reduction of quadratic-bilinear systems," Numerical Linear Algebra with Applications, vol. 25, no. 6, p. e2200, 2018. 
[16] A. Padoan and A. Astolfi, "Model reduction by moment matching at isolated singularities for linear systems: A geometric approach," in 2017 IEEE 56th Annual Conference on Decision and Control (CDC), 2017, pp. 4807-4812.

[17] A. Padoan, G. Scarciotti, and A. Astolfi, "A geometric characterisation of the persistence of excitation condition for the solutions of autonomous systems," To appear on IEEE Transactions on Automatic Control, 2017.

[18] T. C. Ionescu and A. Astolfi, "Nonlinear moment matching-based model order reduction," IEEE Transactions on Automatic Control, vol. 61, no. 10, pp. 2837-2847, 2016.

[19] G. Scarciotti and A. Astolfi, "Model reduction of neutral linear and nonlinear time-invariant time-delay systems with discrete and distributed delays," IEEE Transactions on Automatic Control, vol. 61, no. 6, pp. 1438-1451, 2016.

[20] —, "Model reduction by matching the steady-state response of explicit signal generators," IEEE Transactions on Automatic Control, vol. 61, no. 7, pp. 1995-2000, 2016.

[21] T. C. Ionescu and A. Astolfi, "Families of moment matching based, structure preserving approximations for linear port Hamiltonian systems," Automatica, vol. 49, pp. 2424-2434, 2013.

[22] G. Scarciotti, "Model reduction of power systems with preservation of slow and poorly damped modes," in IEEE Power \& Energy Society General Meeting, Denver, Colorado, July 26-30, 2015, pp. 1-5.

[23] G. Scarciotti and A. Astolfi, "Moment-based discontinuous phasor transform and its application to the steady-state analysis of inverters and wireless power transfer systems," IEEE Transactions on Power Electronics, vol. 31, no. 12, pp. 8448-8460, 2016.

[24] - "Data-driven model reduction by moment matching for linear and nonlinear systems," Automatica, vol. 79, pp. 340-351, May 2017.

[25] G. Scarciotti, Z. P. Jiang, and A. Astolfi, "Data-driven constrained optimal model reduction," European Journal of Control, 2019.

[26] G. Scarciotti and A. Astolfi, "Characterization of the moments of a linear system driven by explicit signal generators," in Proceedings of the 2015 American Control Conference, Chicago, IL, July 1-3, 2015, pp. 589-594.

[27] _ "Model reduction for hybrid systems with state-dependent jumps," in IFAC Symposium Nonlinear Control Systems, Monterey, CA, USA, 2016, pp. 862-867.

[28] G. Scarciotti and A. R. Teel, "On moment matching for stochastic systems," IEEE Transactions on Automatic Control, 2020, conditionally accepted

[29] A. Astolfi, "Model reduction by moment matching for linear and nonlinear systems," IEEE Transactions on Automatic Control, vol. 55, no. 10, pp. 2321-2336, 2010.

[30] J. D. Simard and A. Astolfi, "An interconnection-based interpretation of the Loewner matrices," in 2019 IEEE 58th Conference on Decision and Control (CDC), 2019, pp. 7788-7793.

[31] — "Loewner functions for linear time-varying systems with applications to model reduction," in Proceedings of the 21st IFAC World Congress, 2020, to appear.

[32] _ "Nonlinear model reduction in the Loewner framework," submitted to Transactions on Automatic Control, 2020.

[33] A. Antoulas, J. Ball, J. Kang, and J. Willems, "On the solution of the minimal rational interpolation problem," Linear Algebra and its Applications, vol. 137, pp. 511-573, 1990.

[34] A. C. Antoulas and S. Lefteriu, "A tutorial introduction to the Loewner framework for model reduction," Model Reduction and Approximation: Theory and Algorithms, vol. 15, p. 335, 2017.

[35] T. Ionescu, "Two-sided time-domain moment matching for linear systems," IEEE Transactions on Automatic Control, vol. 61, no. 9, pp. 2632-2637, 2015.

[36] A. J. Krener and M. Xiao, "Nonlinear observer design in the Siegel domain," SIAM Journal on Control and Optimization, vol. 41, no. 3, pp. 932-953, 2002.

[37] J. Carr, Applications of centre manifold theory. Springer Science \& Business Media, 2012, vol. 35.

[38] J. Falnes, Ocean waves and oscillating systems: linear interactions including wave-energy extraction. Cambridge University Press, 2002

[39] W. Cummins, "The impulse response function and ship motions," DTIC Document, Tech. Rep., 1962.

[40] A.-M. Wazwaz, "Volterra integro-differential equations," in Linear and Nonlinear Integral Equations. Springer, 2011, pp. 175-212.
[41] A. Mérigaud and J. V. Ringwood, "Free-surface time-series generation for wave energy applications," IEEE Journal of Oceanic Engineering, vol. 43, no. 1, pp. 19-35, 2018.

[42] N. Faedo, G. Scarciotti, A. Astolfi, and J. V. Ringwood, "Moment-based constrained optimal control of an array of wave energy converters," in 2019 American Control Conf. (ACC), Philadelphia, 2019, pp. 47974802.

[43] K. Hasselmann, "Measurements of wind wave growth and swell decay during the Joint North Sea Wave Project (JONSWAP)," Dtsch. Hydrogr. Z., vol. 8, p. 95, 1973.

[44] N. Faedo, G.-V. Demian, Y. Peña-Sanchez, and J. V. Ringwood, "Optimisation- vs. non-optimisation- based energy-maximising control for wave energy converters," in 2020 European Control Conf. (ECC), St. Petersburg, 2020, pp. 843-848.

[45] J. V. Ringwood, A. Mérigaud, N. Faedo, and F. Fusco, "An analytical and numerical sensitivity and robustness analysis of wave energy control systems," IEEE Trans. on Control Systems Technology, vol. 28, no. 4, pp. 1337-1348, 2020.

[46] N. Faedo, "Optimal control and model reduction for wave energy systems: A moment-based approach," Ph.D. dissertation, Dept. of Electronic Eng., Maynooth University, 2020.

[47] N. Faedo, D. García-Violini, G. Scarciotti, A. Astolfi, and J. V. Ringwood, "Robust moment-based energy-maximising optimal control of wave energy converters," in 58th IEEE Conf. on Decision and Control (CDC), 2019, pp. 4286-4291. 